\title{
Positive solutions of fractional differential equation with two pieces in chain interval and simultaneous Dirichlet boundary conditions
}

\author{
Vahid Hedayati ${ }^{1,2}$ and Mohammad Esmael Samei ${ }^{3^{*}}$ (1)
}

${ }^{*}$ Correspondence:

mesamei@gmail.com;

mesamei@basu.ac.ir

${ }^{3}$ Department of Mathematics,

Faculty of Science, Bu-Ali Sina

University, Hamedan, Iran

Full list of author information is

available at the end of the article

\section{Springer}

\begin{abstract}
In the current study, by using some fixed point technique such as Banach contraction principle and fixed point theorem of Krasnoselskii, we look into the positive solutions for fractional differential equation ${ }^{C} D^{\alpha} u(t)$ equals to $f_{1}\left(t, u(t),{ }^{C} D^{\beta_{1}} u(t), \gamma_{1} u(t)\right)$ and $f_{2}\left(t, u(t),{ }^{C} D^{\beta_{2}} u(t), \gamma_{2} u(t)\right)$ for each $t$ belonging to $\left[0, t_{0}\right]$ and $\left[t_{0}, 1\right]$, respectively, with simultaneous Dirichlet boundary conditions, where ${ }^{c} D^{\alpha}$ and $/^{\alpha}$ denote the Caputo fractional derivative and Riemann-Liouville fractional integral of order $\alpha$, respectively. Some models are thrown to illustrate our results, too.
\end{abstract}

MSC: Primary 34A08; 39A12; secondary 39A13

Keywords: Positive solutions; Fractional differential equation; Dirichlet boundary conditions; Caputo fractional derivative; Riemann-Liouville fractional integral

\section{Introduction}

Fractional calculus is an important branch in mathematical analysis. However, after Leibniz and Newton invented differential calculus, it has been a topic of interest among mathematicians, engineers, and physicists. It is known that fractional calculus has numerous applications in different sciences such as mechanics, electricity, biology, control theory, signal and image processing (for example, see [1-4] and the references therein). In recent years the fractional differential equations and inclusions, in two type differential and q-differential, have been developed intensively (for more details, see [5-27] and the references therein).

It is given that the existence results of fractional differential equation of all articles are presented in a single interval. So, there exists a question as follows: "What is the solution, if the fractional differential equation is defined on a piecewise function or even piecewise multi-function?" In this research, we investigate the positive solutions of fractional differential equation with two pieces in chain interval and simultaneous Dirichlet boundary

(c) The Author(s) 2019. This article is distributed under the terms of the Creative Commons Attribution 4.0 International License (http://creativecommons.org/licenses/by/4.0/), which permits unrestricted use, distribution, and reproduction in any medium, provided you give appropriate credit to the original author(s) and the source, provide a link to the Creative Commons license, and indicate if changes were made. 
conditions as follows:

$$
\begin{aligned}
& { }^{c} D^{\alpha} u(t)= \begin{cases}f_{1}\left(t, u(t),{ }^{c} D^{\beta_{1}} u(t), I^{\gamma_{1}} u(t)\right), & 0 \leq t \leq t_{0}, \\
f_{2}\left(t, u(t),{ }^{c} D^{\beta_{2}} u(t), I^{\gamma_{2}} u(t)\right), & t_{0} \leq t \leq 1,\end{cases} \\
& u(0)=h_{1}\left(t_{0}, u\left(t_{0}\right),{ }^{c} D^{\beta_{3}} u\left(t_{0}\right), I^{\gamma_{3}} u\left(t_{0}\right)\right), \\
& u(1)=h_{2}\left(t_{0}, u\left(t_{0}\right),{ }^{c} D^{\beta_{4}} u\left(t_{0}\right), I^{\gamma_{4}} u\left(t_{0}\right)\right),
\end{aligned}
$$

where $1<\alpha \leq 2$, and ${ }^{c} D^{\alpha}, I^{\alpha}$ denote the Caputo fractional derivative and RiemannLiouville integral of order $\alpha$, respectively, $t \in \bar{J}=[0,1], t_{0} \in J=(0,1), \beta_{i} \in(0,1), \gamma_{i} \in(0, \infty)$, here $i=1,2,3,4$, and the functions $f_{j}$ and $h_{j}$ map $\bar{J} \times \mathbb{R}^{3}$ to $\mathbb{R}$ for $j=1,2$ such that $f_{1}\left(t_{0}, \cdot, \cdot, \cdot\right)=f_{2}\left(t_{0}, \cdot, \cdot, \cdot\right)$.

In 2009, Su and Zhang presented analysis of the boundary value problem for the fractional differential equation involving more general boundary condition and a nonlinear term dependent on the fractional of the unknown function

$$
{ }^{C} D_{0^{+}}^{\alpha} u(t)=T\left(t, u(t),{ }^{C} D_{0^{+}}^{\beta} u(t)\right),
$$

$a_{1} u(0)-a_{2} u^{\prime}(0)=A$, and $b_{1} u(1)+b_{2} u^{\prime}(1)=B$ for all $t \in(0,1)$, where $\alpha \in(1,2], \beta \in(0,1]$, $a_{i}, b_{i} \geq 0$, for $i=1,2$, with $a_{1} b_{1}+a_{1} b_{2}+a_{2} b_{1}>0, T:[0,1] \times \mathbb{R}^{2} \rightarrow \mathbb{R}$ is continuous and ${ }^{C} D_{0^{+}}^{\alpha}$ is the Caputo fractional derivative [5]. In the next year, Ahmad and Sivasundaram proved the existence of solutions for the nonlinear fractional integro-differential equation ${ }^{c} D^{\alpha} u(t)=T\left(t, u(t),\left(\phi_{1} u\right)(t),\left(\phi_{1} u\right)(t)\right)$ for each $t \in(0,1)$, with boundary values $u^{\prime}(0)+a u\left(\eta_{1}\right)=0$ and $b u^{\prime}(1)+u\left(\eta_{2}\right)=0$, where $\alpha \in(1,2], 0<\eta_{1} \leq \eta_{2}<1, a, b \in(0,1)$, the map $T:[0,1] \times X^{3} \rightarrow X$ is continuous and for the map $\gamma_{i}$ maps $[0,1]^{2}$ into $\mathbb{R}^{\geq 0}$ with some properties, the map $\phi_{i}$ is defined by $\left(\phi_{i} u\right)(t)=\int_{0}^{t} \gamma_{i}(t, s) u(s) d s$ [6]. In 2011, Agarwal, Regan, and Staněk investigated the singular fractional mixed boundary value problem

$$
{ }^{c} D^{\alpha} f(x)+T\left(x, f(x), f^{\prime}(x),{ }^{c} D^{\mu} f(x)\right)=0,
$$

$f(1)=f^{\prime}(0)=0$ for all $t \in[0,1]$, where $\mu \in(0,1),{ }^{c} D^{\alpha}$ is the Caputo fractional derivative of order $\alpha$ with $\alpha \in(1,2)$, the positive function $T$ is a scaler $L^{\kappa}$-Carathéodory on [0.1] $\times E$ with $E=(0, \infty) \times(0, \infty) \times(0, \infty)$, and $\kappa>\frac{1}{\alpha-1}$ such that $T\left(t, x_{1}, x_{2}, x_{3}\right)$ may be singular at the value 0 in one dimension of its space variables $x_{1}, x_{2}, x_{3}$ [7].

In 2013, Baleanu, Rezapour, and Mohammadi discussed the nonlinear fractional differential equation ${ }^{c} D^{\alpha} x(t)=f(t, x(t))$ with the integral boundary condition $x(0)=0$, and $x(1)=\int_{0}^{\eta} s(s) d s$ for $0<t, \eta<1$, and $\alpha \in(1,2]$, where ${ }^{c} D^{\alpha}$ denotes the Caputo fractional derivative of order $\alpha$ and $f$ maps [0,1] $\times X$ into $X$ is a continuous function [8]. Also, they studied the existence of solutions for the singular nonlinear fractional boundary value problem

$$
\left\{\begin{array}{l}
{ }^{c} D^{\alpha} y(x)=T\left(x, y(x), y^{\prime}(x),{ }^{c} D^{\beta} y(x)\right), \\
y(0)=a y(1), \quad y^{\prime}(0)=b^{c} D^{\beta} y(1), \quad y^{\prime \prime}(0)=y^{\prime \prime \prime}(0)=y^{(n-1)}(0)=0,
\end{array}\right.
$$

where number $n$ more than or equal to three is an integer, $\alpha$ in $(n-1, n), 0<\beta<1,0<a<1$, $0<b<\Gamma(2-\beta), T$ is an $L^{q}$-Carathéodory function, $q(\alpha-1)>1$, and $T\left(t, y_{1}, y_{2}, y_{3}\right)$ may be 
singular at value 0 in one dimension of its space variables $y_{1}, y_{2}$, and $y_{3}$ [9]. In addition to that, in the same year, Baleanu, Nazemi, and Rezapour studied the multi-term nonlinear fractional integro-differential equations

$$
\left\{\begin{array}{l}
{ }^{c} D^{\alpha} f(t)=T\left(t, f(t),(\phi f)(t),(\psi f)(t),{ }^{c} D^{\beta_{1}} f(t),{ }^{c} D^{\beta_{2}} f(t), \ldots,{ }^{c} D^{\beta_{n}} f(t)\right), \\
u(0)+a u(1)=0, \quad u^{\prime}(0)+b u^{\prime}(1)=0,
\end{array}\right.
$$

for each $t \in(0,1)$, where $\alpha \in(1,2), \beta_{i} \in(0,1)$, when $i=1, \ldots, n$ with $\alpha-\beta_{i} \geq 1, a, b \neq-1$, function $f$ maps $\bar{J} \times \mathbb{R}^{n+3}$ into $\mathbb{R}$ is continuous, and the mappings $\phi$ and $\psi$ with the same characteristic as Agarwal in 2010 [10]. One year later, in 2014, Agarwal et al. analyzed the fractional derivative inclusion ${ }^{c} D^{q} x(t) \in F\left(t, x(t),{ }^{c} D^{\beta} x(t)\right)$ for all $t \in \bar{J}$, with conditions $x(1)+x^{\prime}(1)=\int_{0}^{\eta} x(s) d s$ and $x(0)=0$, where $\beta, \eta \in(0,1), q \in(1,2]$ with $q-\beta>1$ and $F$ : $J \times \mathbb{R}^{2} \rightarrow 2^{\mathbb{R}}$ denotes a compact-valued multifunction [11].

In 2016, Bachar, Mâagli, and Rădulescu studied the fractional Navier boundary value problem $D^{\alpha}\left(D^{\beta} u\right)(x)=u(x) f(x, u(x))=0$ for $x \in(0,1)$ with conditions $\lim _{x \rightarrow 0^{+}} D^{\beta-1} u(x)=$ $0, \lim _{x \rightarrow 0^{+}} D^{\alpha-1}\left(D^{\beta} u\right)(x)=\eta_{1}, u(1)=0$, and $D^{\beta} u(1)=-\eta_{2}$, where $\alpha, \beta \in(1,2], D^{\alpha}$ and $D^{\beta}$ stand for the standard Riemann-Liouville fractional derivatives and $\eta_{i} \in[0, \infty)$ are somehow that $\eta_{1}+\eta_{2} \in(0, \infty)$ [28]. Also, in the same year, Zhang and Zhong founded the multiplicity of positive solutions for the nonlocal singular fractional differential equations $D_{0^{+}}^{\alpha} f(t)+T(t, f(t))=0$, with boundary value $f(0)=D_{0^{+}}^{\beta} f(0)=0$, and $D_{0^{+}}^{\beta} f(1)=$ $\sum_{i=1}^{\infty} \xi_{i} D_{0^{+}}^{\beta} f\left(\eta_{i}\right)$ for almost all $t \in(0,1)$, where $\alpha \in(2,3], \beta \in[1,2], 0<\xi_{i}, \eta_{i}<1$ with $\sum_{i=1}^{\infty} \xi_{i} \eta_{i}^{\alpha-\beta-1}<1, f$ belongs to $C((0,1) \times(0, \infty),[0, \infty))$, and $D_{0^{+}}^{\alpha}$ is the standard RiemannLiouville fractional derivative of order $\alpha$ [12]. Then, in 2017, Rezapour and Hedayati investigated the existence of solutions for the Caputo fractional differential inclusion

$$
{ }^{c} D^{\alpha} x(t) \in T\left(t, x(t),{ }^{c} D^{\beta} x(t), x^{\prime}(t)\right)
$$

for each $t \in[0,1]$ via the integral boundary value conditions $x(0)+x^{\prime}(0)+{ }^{c} D^{\beta} x(0)=$ $\int_{0}^{\eta} x(s) d s$ and $x(1)+x^{\prime}(1)+{ }^{c} D^{\beta} x(1)=\int_{0}^{v} x(s) d s$, where $T:[0,1] \times \mathbb{R}^{3} \rightarrow 2^{\mathbb{R}}$ is a compactvalued multifunction and ${ }^{c} D^{\alpha}$ is the Caputo differential operator of order $\alpha \in(1,2]$ [13]. In the same year, Denton and Ramírez consider integro-differential initial value problems $D^{q} u(t)=f(t, u(t), T u(t))+g(t, u(t), T u(t))$ with $\left.u(t)(t-a)^{p}\right|_{t=a}=u^{0}$, where $t \in J=[0,1]$, the functions $f, g$ belong to $\left.C U \times \mathbb{R}^{2}, \mathbb{R}\right], T u(t)=\int_{0}^{t} K(t, s) u(s) d s$ here $K \in C\left(J^{2}, \mathbb{R}\right)$ and $D^{q}$ Riemann-Liouville fractional derivatives and the forcing function is a sum of an increasing function and a decreasing function [29].

In 2018, Aydogan et al. gave a new method to investigate some fractional integrodifferential equations involving the Caputo-Fabrizio derivation [14]. In addition, in the next article, Baleanu, Mousalou, and Rezapour extended fractional Caputo-Fabrizio derivative for the existence of solutions for two higher-order series-type differential equations [15]. Besides that, Chidouh and Torres proved some generalizations of the Lyapunov inequality for the following discrete fractional boundary value problem:

$$
\begin{cases}\Delta^{\alpha} y+q(t+\alpha-1) f(y(t+\alpha-1))=0, & \alpha \in(1,2], \\ y(\alpha-2)=y(\alpha+b+1)=0, & b \in[2, \infty),\end{cases}
$$


where $b \in \mathbb{N}$ and $\Delta^{\alpha}$ is an operator with some properties [30]. Also, in 2019, Samei and Khalilzadeh Ranjbar discussed the fractional hybrid q-differential inclusions

$$
{ }^{c} D_{q}^{\alpha}\left(x / f\left(t, x, I_{q}^{\alpha_{1}} x, \ldots, I_{q}^{\alpha_{n}} x\right)\right) \in F\left(t, x, I_{q}^{\beta_{1}} x, \ldots, I_{q}^{\beta_{k}} x\right),
$$

with the boundary conditions $x(0)=x_{0}$ and $x(1)=x_{1}$, where $1<\alpha \leq 2, q \in(0,1), x_{0}, x_{1} \in$ $\mathbb{R}, \alpha_{i}>0$, for $i=1,2, \ldots, n, \beta_{j}>0$, for $j=1,2, \ldots, k, n, k \in \mathbb{N},{ }^{c} D_{q}^{\alpha}$ denotes Caputo type qderivative of order $\alpha, I_{q}^{\beta}$ denotes Riemann-Liouville type q-integral of order $\beta, f: J \times$ $\mathbb{R}^{n} \rightarrow(0, \infty)$ is continuous, and $F$ maps $J \times \mathbb{R}^{k}$ to $P(\mathbb{R})$ is multifunction [16]. Liu presented a new method for converting boundary value problems of impulsive fractional differential equations to integral equations and gave the method for applications [31].

\section{Preliminaries}

Here, we recall some basic notion, lemmas, and theorems which are used in the subsequent sections.

Definition 1 The Riemann-Liouville fractional integral of order $\alpha>0$ for a function $y$ is defined by

$$
I_{a}^{\alpha} y(t)=\frac{1}{\Gamma(\alpha)} \int_{a}^{t}(t-s)^{\alpha-1} y(s) d s .
$$

In particular, $I_{0}^{\alpha} y(t):=I^{\alpha} y(t)$.

Definition 2 Let $n \in \mathbb{N}, n-1<\alpha \leq n, y \in A C^{n}[a, b]$, where $0 \leq a<b<\infty$ and

$$
A C^{n}[a, b]=\left\{y:[a, b] \rightarrow \mathbb{R}: \frac{d^{n} y(t)}{d t^{n}} \in A C[a, b]\right\} .
$$

(i) If $\alpha \neq n$, then the Caputo fractional derivative of order $\alpha$ is defined by

$$
{ }^{c} D_{a}^{\alpha} y(t)=\frac{1}{\Gamma(n-\alpha)} \int_{a}^{t}(t-s)^{n-\alpha-1} y^{(n)}(s) d s=I_{a}^{n-\alpha} y^{(n)}(s) .
$$

(ii) If $\alpha=n$, then the Caputo fractional derivative of order $n$ is defined by

$$
{ }^{c} D_{a}^{n} y(t)=y^{(n)}(t)
$$

In particular, ${ }^{c} D_{0}^{0} y(t)=y(t),{ }^{c} D_{0}^{\alpha} y(t)={ }^{c} D^{\alpha} y(t)$.

Lemma 3 ([3]) Let $n \in \mathbb{N}, n-1<\alpha \leq n$, and $y \in A C^{n}[a, b]$. Then one has

$$
I_{a}^{\alpha}\left({ }^{c} D_{a}^{\alpha}\right) y(t)=y(t)+\sum_{i=0}^{n-1} c_{i}(t-a)^{i},
$$

where $c_{0}, c_{1}, \ldots, c_{n-1} \in \mathbb{R}$.

Lemma 4 ([3]) Let $n \in \mathbb{N}, n-1<\alpha \leq n$, and $y \in C[a, b]$. Then one has ${ }^{c} D_{a}^{\alpha}\left(I_{a}^{\alpha}\right) y(t)=y(t)$. 
Lemma 5 ([3]) Let $\alpha \in(0,1)$. Then, for each $y \in A C[0,1], I^{\alpha} D^{\alpha} y(t)=y(t)$ for almost everywhere $t \in[0,1]$, where

$$
D^{\alpha} y(t)=\frac{1}{\Gamma(1-\alpha)} \frac{d}{d t}\left(\int_{0}^{t}(t-s)^{-\alpha} y(s) d s\right)
$$

The following fixed point theorems are used in the next section.

Theorem 6 ([32] Banach contraction principle) Let $X$ be a Banach space. If $A: X \rightarrow X$ is the contraction map, then there exists $x \in X$ such that $A x=x$.

Theorem 7 ([32] Krasnoselskii's fixed point theorem) Let $C$ be a closed convex and nonempty subset of a Banach space $\mathcal{X}$. Suppose that $F_{1}$ and $F_{2}$ are two maps of $C$ into $\mathcal{X}$ such that $F_{1} x+F_{2} y \in C$ for each $x, y \in C$. If $F_{1}$ is a compact and continuous map and $F_{2}$ is a contraction map, then there exists $x \in C$ such that $x=F_{1} x+F_{2} x$.

\section{Main results}

In this section, we examine the existence of solution for boundary value problem (1).

Lemma 8 The unique solution of the fractional differential equation ${ }^{c} D^{\alpha} u(t)=v(t)$ with the boundary conditions $u(0)=h_{1}\left(t_{0}, u\left(t_{0}\right),{ }^{c} D^{\beta_{3}} u\left(t_{0}\right), I^{\gamma_{3}} u\left(t_{0}\right)\right), u(1)=h_{2}\left(t_{0}, u\left(t_{0}\right),{ }^{c} D^{\beta_{4}} u\left(t_{0}\right)\right.$, $\left.I^{\gamma_{4}} u\left(t_{0}\right)\right)$ is

$$
\begin{aligned}
u(t)= & \frac{1}{\Gamma(\alpha)} \int_{0}^{t}(t-s)^{\alpha-1} v(s) d s+h_{1}\left(t_{0}, u\left(t_{0}\right),{ }^{c} D^{\beta_{3}} u\left(t_{0}\right), I^{\gamma_{3}} u\left(t_{0}\right)\right) \\
& +\left[h_{2}\left(t_{0}, u\left(t_{0}\right),{ }^{c} D^{\beta_{4}} u\left(t_{0}\right), I^{\gamma_{4}} u\left(t_{0}\right)\right)-\frac{1}{\Gamma(\alpha)} \int_{0}^{1}(1-s)^{\alpha-1} v(s) d s\right. \\
& \left.-h_{1}\left(t_{0}, u\left(t_{0}\right),{ }^{c} D^{\beta_{3}} u\left(t_{0}\right), I^{\gamma_{3}} u\left(t_{0}\right)\right)\right] t,
\end{aligned}
$$

where $v \in L^{1}(\bar{J}, \mathbb{R})$ and $u \in A C^{2}(\bar{J}, \mathbb{R})$.

Proof Assume that $u(t)$ is a solution of equation ${ }^{c} D^{\alpha} u(t)=v(t)$. By using Lemma 3 and properties of the operator $I^{\alpha}$, we obtain $u(t)=I^{\alpha} v(t)+c_{0}+c_{1} t$, where $c_{0}, c_{1} \in \mathbb{R}$ denote arbitrary constants. Now, by applying the boundary conditions, we get $c_{0}=$ $h_{1}\left(t_{0}, u\left(t_{0}\right),{ }^{c} D^{\beta_{3}} u\left(t_{0}\right), I^{\gamma_{3}} u\left(t_{0}\right)\right)$ and

$$
\begin{aligned}
c_{1}= & h_{2}\left(t_{0}, u\left(t_{0}\right),{ }^{c} D^{\beta_{4}} u\left(t_{0}\right), I^{\gamma_{4}} u\left(t_{0}\right)\right) \\
& -\frac{1}{\Gamma(\alpha)} \int_{0}^{1}(1-s)^{\alpha-1} v(s) d s-h_{1}\left(t_{0}, u\left(t_{0}\right),{ }^{c} D^{\beta_{3}} u\left(t_{0}\right), I^{\gamma_{3}} u\left(t_{0}\right)\right) .
\end{aligned}
$$

Conversely, by simple check, we conclude that equation (2) satisfies the boundary conditions

$$
\begin{aligned}
& u(0)=h_{1}\left(t_{0}, u\left(t_{0}\right),{ }^{c} D^{\beta_{3}} u\left(t_{0}\right), I^{\gamma_{3}} u\left(t_{0}\right)\right), \\
& u(1)=h_{2}\left(t_{0}, u\left(t_{0}\right),{ }^{c} D^{\beta_{4}} u\left(t_{0}\right), I^{\gamma_{4}} u\left(t_{0}\right)\right) .
\end{aligned}
$$


It is obvious that Lemmas 4 and 5 imply that

$$
{ }^{c} D^{\alpha} x(t)=I^{2-\alpha}\left(x^{\prime \prime}(t)\right)=I^{2-\alpha}\left(I^{-2+\alpha} v(t)\right)=I^{2-\alpha}\left(D^{2-\alpha} v(t)\right)=v(t) .
$$

This completes our proof.

Consider the space $\mathcal{X}=C^{1}(\bar{J}, \mathbb{R})$ with the norm $\|x\|_{*}=\|x\|+\left\|x^{\prime}\right\|$, where $\|x\|=$ $\sup \{|x(t)|, t \in J\}$.

Corollary 1 A function $u \in \mathcal{X}$ is a solution of problem (1) if and only if

$$
\begin{aligned}
u(t)= & \frac{1}{\Gamma(\alpha)} \int_{0}^{t}(t-s)^{\alpha-1} f_{1}\left(s, u(s),{ }^{c} D^{\beta_{1}} u(s), I^{\gamma_{1}} u(s)\right) d s \\
& +h_{1}\left(t_{0}, u\left(t_{0}\right),{ }^{c} D^{\beta_{3}} u\left(t_{0}\right), I^{\gamma_{3}} u\left(t_{0}\right)\right)+\Delta_{u}\left(t_{0}\right) t
\end{aligned}
$$

whenever $0 \leq t \leq t_{0}$, and

$$
\begin{aligned}
u(t)= & \frac{1}{\Gamma(\alpha)} \int_{0}^{t_{0}}(t-s)^{\alpha-1} f_{1}\left(s, u(s),{ }^{c} D^{\beta_{1}} u(s), I^{\gamma_{1}} u(s)\right) d s \\
& +\frac{1}{\Gamma(\alpha)} \int_{t_{0}}^{t}(t-s)^{\alpha-1} f_{2}\left(s, u(s),{ }^{c} D^{\beta_{2}} u(s), I^{\gamma_{2}} u(s)\right) d s \\
& +h_{1}\left(t_{0}, u\left(t_{0}\right),{ }^{c} D^{\beta_{3}} u\left(t_{0}\right), I^{\gamma_{3}} u\left(t_{0}\right)\right)+\Delta_{u}\left(t_{0}\right) t,
\end{aligned}
$$

whenever $t_{0} \leq t \leq 1$, here

$$
\begin{aligned}
\Delta_{u}\left(t_{0}\right)= & h_{2}\left(t_{0}, u\left(t_{0}\right),{ }^{c} D^{\beta_{4}} u\left(t_{0}\right), I^{\gamma_{4}} u\left(t_{0}\right)\right) \\
& -\frac{1}{\Gamma(\alpha)} \int_{0}^{t_{0}}(1-s)^{\alpha-1} f_{1}\left(s, u(s),{ }^{c} D^{\beta_{1}} u(s), I^{\gamma_{1}} u(s)\right) d s \\
& -\frac{1}{\Gamma(\alpha)} \int_{t_{0}}^{1}(1-s)^{\alpha-1} f_{2}\left(s, u(s),{ }^{c} D^{\beta_{2}} u(s), I^{\gamma_{2}} u(s)\right) d s \\
& -h_{1}\left(t_{0}, u\left(t_{0}\right),{ }^{c} D^{\beta_{3}} u\left(t_{0}\right), I^{\gamma_{3}} u\left(t_{0}\right)\right) .
\end{aligned}
$$

Theorem 9 Problem (1) has a unique solution whenever there exist $k$ belonging to $(0, \alpha-1)$ and $\gamma_{i}, \mu_{i}$ in $\in L^{\frac{1}{k}}(\bar{J},(0, \infty)), C(\bar{J},(0, \infty))$, respectively, for $i=1,2$, such that

$$
\begin{aligned}
& \left|f_{1}\left(t, x_{1}, x_{2}, x_{3}\right)-f_{1}\left(t, x_{1}^{\prime}, x_{2}^{\prime}, x_{3}^{\prime}\right)\right| \leq \mu_{1}(t) \sum_{j=1}^{3}\left|x_{j}-x_{j}^{\prime}\right|, \\
& \left|f_{2}\left(t, x_{1}, x_{2}, x_{3}\right)-f_{2}\left(t, x_{1}^{\prime}, x_{2}^{\prime}, x_{3}^{\prime}\right)\right| \leq \mu_{2}(t) \sum_{j=1}^{3}\left|x_{j}-x_{j}^{\prime}\right|, \\
& \left|h_{1}\left(t, x_{1}, x_{2}, x_{3}\right)-h_{1}\left(t, x_{1}^{\prime}, x_{2}^{\prime}, x_{3}^{\prime}\right)\right| \leq v_{1}(t) \sum_{j=1}^{3}\left|x_{j}-x_{j}^{\prime}\right|, \\
& \left|h_{2}\left(t, x_{1}, x_{2}, x_{3}\right)-h_{2}\left(t, x_{1}^{\prime}, x_{2}^{\prime}, x_{3}^{\prime}\right)\right| \leq v_{2}(t) \sum_{j=1}^{3}\left|x_{j}-x_{j}^{\prime}\right|,
\end{aligned}
$$


and

$$
\begin{aligned}
\Lambda= & \frac{3\left\|\mu_{1}\right\|_{\frac{1}{k}}}{\Gamma(\alpha)}\left[1+\frac{1}{\Gamma\left(2-\beta_{1}\right)}+\frac{1}{\Gamma\left(1+\gamma_{1}\right)}\right]\left(\frac{1-k}{\alpha-k}\right)^{1-k} \\
& +\frac{3\left\|\mu_{2}\right\|_{\frac{1}{k}}}{\Gamma(\alpha)}\left[1+\frac{1}{\Gamma\left(2-\beta_{2}\right)}+\frac{1}{\Gamma\left(1+\gamma_{2}\right)}\right]\left(\frac{1-k}{\alpha-k}\right)^{1-k} \\
& +3\left\|v_{1}\right\|\left[1+\frac{1}{\Gamma\left(2-\beta_{3}\right)}+\frac{1}{\Gamma\left(1+\gamma_{3}\right)}\right] \\
& +2\left\|v_{2}\right\|\left[1+\frac{1}{\Gamma\left(2-\beta_{4}\right)}+\frac{1}{\Gamma\left(1+\gamma_{4}\right)}\right] \\
& +\frac{\left\|\mu_{1}\right\|_{\frac{1}{k}}}{\Gamma(\alpha-1)}\left[1+\frac{1}{\Gamma\left(2-\beta_{1}\right)}+\frac{1}{\Gamma\left(1+\gamma_{1}\right)}\right]\left(\frac{1-k}{\alpha-k+1}\right)^{1-k} \\
& +\frac{\left\|\mu_{2}\right\|_{\frac{1}{k}}}{\Gamma(\alpha-1)}\left[1+\frac{1}{\Gamma\left(2-\beta_{2}\right)}+\frac{1}{\Gamma\left(1+\gamma_{2}\right)}\right]\left(\frac{1-k}{\alpha-k+1}\right)^{1-k}
\end{aligned}
$$

$<1$

for all $t \in \bar{J}, x_{j}, x_{j}^{\prime} \in \mathbb{R}(i=1,2,3)$, here $\|L\|_{p}=\left(\int_{0}^{1}|L(s)|^{p} d s\right)^{\frac{1}{p}}$ for all $L$ belongs to $L^{p}(J, \mathbb{R})$.

Proof Define the operator $N: \mathcal{X} \rightarrow \mathcal{X}$ by

$$
\begin{aligned}
N u(t)= & \frac{1}{\Gamma(\alpha)} \int_{0}^{t}(t-s)^{\alpha-1} f_{1}\left(s, u(s),{ }^{c} D^{\beta_{1}} u(s), I^{\gamma_{1}} u(s)\right) d s \\
& +h_{1}\left(t_{0}, u\left(t_{0}\right),{ }^{c} D^{\beta_{3}} u\left(t_{0}\right), I^{\gamma_{3}} u\left(t_{0}\right)\right)+\Delta_{u}\left(t_{0}\right) t
\end{aligned}
$$

whenever $0 \leq t \leq t_{0}$, and

$$
\begin{aligned}
N u(t)= & \frac{1}{\Gamma(\alpha)} \int_{0}^{t_{0}}(t-s)^{\alpha-1} f_{1}\left(s, u(s),{ }^{c} D^{\beta_{1}} u(s), I^{\gamma_{1}} u(s)\right) d s \\
& +\frac{1}{\Gamma(\alpha)} \int_{t_{0}}^{t}(t-s)^{\alpha-1} f_{2}\left(s, u(s),{ }^{c} D^{\beta_{2}} u(s), I^{\gamma_{2}} u(s)\right) d s \\
& +h_{1}\left(t_{0}, u\left(t_{0}\right),{ }^{c} D^{\beta_{3}} u\left(t_{0}\right), I^{\gamma_{3}} u\left(t_{0}\right)\right)+\Delta_{u}\left(t_{0}\right) t,
\end{aligned}
$$

whenever $t_{0} \leq t \leq 1$. It is easy to check that problem (1) has solutions if and only if the operator equation $N u=u$ has fixed points. Let $u, v \in \mathcal{X}$. If $0 \leq t \leq t_{0}$, then we obtain

$$
\begin{aligned}
|N u(t)-N v(t)|= & \mid \frac{1}{\Gamma(\alpha)} \int_{0}^{t}(t-s)^{\alpha-1} f_{1}\left(s, u(s),{ }^{c} D^{\beta_{1}} u(s), I^{\gamma_{1}} u(s)\right) d s \\
& +h_{1}\left(t_{0}, u\left(t_{0}\right),{ }^{c} D^{\beta_{3}} u\left(t_{0}\right), I^{\gamma_{3}} u\left(t_{0}\right)\right)+\Delta_{u}\left(t_{0}\right) t \\
& -\frac{1}{\Gamma(\alpha)} \int_{0}^{t}(t-s)^{\alpha-1} f_{1}\left(s, v(s),{ }^{c} D^{\beta_{1}} v(s), I^{\gamma_{1}} v(s)\right) d s \\
& -h_{1}\left(t_{0}, v\left(t_{0}\right),{ }^{c} D^{\beta_{3}} v\left(t_{0}\right), I^{\gamma_{3}} f\left(t_{0}\right)\right)-\Delta_{v}\left(t_{0}\right) t \mid \\
\leq & \frac{1}{\Gamma(\alpha)} \int_{0}^{t}(t-s)^{\alpha-1} \mid f_{1}\left(s, u(s),{ }^{c} D^{\beta_{1}} u(s), I^{\gamma_{1}} u(s)\right)
\end{aligned}
$$




$$
\begin{aligned}
& -f_{1}\left(s, v(s),{ }^{c} D^{\beta_{1}} v(s), I^{\gamma_{1}} v(s)\right) \mid d s \\
& +2 \mid h_{1}\left(t_{0}, u\left(t_{0}\right),{ }^{c} D^{\beta_{3}} u\left(t_{0}\right), I^{\gamma_{3}} u\left(t_{0}\right)\right) \\
& -h_{1}\left(t_{0}, v\left(t_{0}\right),{ }^{c} D^{\beta_{3}} v\left(t_{0}\right), I^{\gamma_{3}} v\left(t_{0}\right)\right) \\
& +\mid h_{2}\left(t_{0}, u\left(t_{0}\right),{ }^{c} D^{\beta_{4}} u\left(t_{0}\right), I^{\gamma_{4}} u\left(t_{0}\right)\right) \\
& -h_{2}\left(t_{0}, v\left(t_{0}\right),{ }^{c} D^{\beta_{4}} v\left(t_{0}\right), I^{\gamma_{4}} v\left(t_{0}\right)\right) \\
& +\frac{1}{\Gamma(\alpha)} \int_{0}^{t_{0}}(1-s)^{\alpha-1} \mid f_{1}\left(s, u(s),{ }^{c} D^{\beta_{1}} u(s), I^{\gamma_{1}} u(s)\right) \\
& -f_{1}\left(s, v(s),{ }^{c} D^{\beta_{1}} v(s), I^{\gamma_{1}} v(s)\right) \mid d s \\
& +\frac{1}{\Gamma(\alpha)} \int_{t_{0}}^{1}(1-s)^{\alpha-1} \mid f_{2}\left(s, u(s),{ }^{c} D^{\beta_{2}} u(s), I^{\gamma_{2}} u(s)\right) \\
& -f_{2}\left(s, v(s),{ }^{c} D^{\beta_{2}} v(s), I^{\gamma_{2}} v(s)\right) \mid d s \\
& \leq \frac{1}{\Gamma(\alpha)} \int_{0}^{t}(t-s)^{\alpha-1} \mu_{1}(s)(|u(s)-v(s)| \\
& \left.+\left|{ }^{c} D^{\beta_{1}} u(s)-{ }^{c} D^{\beta_{1}} v(s)\right|+\left|I^{\gamma_{1}} u(s)-I^{\gamma_{1}} v(s)\right|\right) d s \\
& +2 v_{1}\left(t_{0}\right)\left(\left|u\left(t_{0}\right)-v\left(t_{0}\right)\right|\right. \\
& \left.+\left|{ }^{c} D^{\beta_{3}} u\left(t_{0}\right)-{ }^{c} D^{\beta_{3}} v\left(t_{0}\right)\right|+\left|I^{\gamma_{3}} v\left(t_{0}\right)-I^{\gamma_{3}} v\left(t_{0}\right)\right|\right) \\
& +v_{2}\left(t_{0}\right)\left(\left|u\left(t_{0}\right)-v\left(t_{0}\right)\right|+\left|{ }^{c} D^{\beta_{4}} u\left(t_{0}\right)-{ }^{c} D^{\beta_{4}} v\left(t_{0}\right)\right|\right. \\
& \left.+\left|I^{\gamma_{4}} u\left(t_{0}\right)-I^{\gamma_{4}} v\left(t_{0}\right)\right|\right) \\
& +\frac{1}{\Gamma(\alpha)} \int_{0}^{t_{0}}(1-s)^{\alpha-1} \mu_{1}(s)(|u(s)-v(s)| \\
& \left.+\left|{ }^{c} D^{\beta_{1}} u(s)-{ }^{c} D^{\beta_{1}} v(s)\right|+\left|I^{\gamma_{1}} u(s)-I^{\gamma_{1}} v(s)\right|\right) d s \\
& +\frac{1}{\Gamma(\alpha)} \int_{t_{0}}^{1}(1-s)^{\alpha-1} \mu_{2}(s)(|u(s)-v(s)| \\
& \left.+\left|{ }^{c} D^{\beta_{2}} u(s)-{ }^{c} D^{\beta_{2}} v(s)\right|+\left|I^{\gamma_{1}} u(s)-I^{\gamma_{2}} v(s)\right|\right) d s \\
& \leq \frac{1}{\Gamma(\alpha)} \int_{0}^{t}(t-s)^{\alpha-1} \mu_{1}(s)(|u(s)-v(s)| \\
& +\frac{1}{\Gamma\left(1-\beta_{1}\right)} \int_{0}^{s}(s-\tau)^{-\beta_{1}}\left|u^{\prime}(\tau)-v^{\prime}(\tau)\right| d \tau \\
& \left.+\frac{1}{\Gamma\left(\gamma_{1}\right)} \int_{0}^{s}(s-\tau)^{\gamma_{1}-1}|u(\tau)-v(\tau)| d \tau\right) d s \\
& +2 v_{1}\left(t_{0}\right)\left(\left|u\left(t_{0}\right)-v\left(t_{0}\right)\right|\right. \\
& +\frac{1}{\Gamma\left(1-\beta_{3}\right)} \int_{0}^{t_{0}}\left(t_{0}-\tau\right)^{-\beta_{3}}\left|u^{\prime}(\tau)-v^{\prime}(\tau)\right| d \tau \\
& \left.+\frac{1}{\Gamma\left(\gamma_{3}\right)} \int_{0}^{t_{0}}\left(t_{0}-\tau\right)^{\gamma_{3}-1}|u(\tau)-v(\tau)| d \tau\right) \\
& +v_{2}\left(t_{0}\right)\left(\left|u\left(t_{0}\right)-v\left(t_{0}\right)\right|\right.
\end{aligned}
$$




$$
\begin{aligned}
& +\frac{1}{\Gamma\left(1-\beta_{4}\right)} \int_{0}^{t_{0}}\left(t_{0}-\tau\right)^{-\beta_{4}}\left|u^{\prime}(\tau)-v^{\prime}(\tau)\right| d \tau \\
& \left.+\frac{1}{\Gamma\left(\gamma_{4}\right)} \int_{0}^{t_{0}}\left(t_{0}-\tau\right)^{\gamma_{4}-1}|u(\tau)-v(\tau)| d \tau\right) \\
& +\frac{1}{\Gamma(\alpha)} \int_{0}^{t_{0}}(1-s)^{\alpha-1} \mu_{1}(s)(|u(s)-v(s)| \\
& +\frac{1}{\Gamma\left(1-\beta_{1}\right)} \int_{0}^{s}(s-\tau)^{-\beta_{1}}\left|u^{\prime}(u)-v^{\prime}(u)\right| d \tau \\
& \left.+\frac{1}{\Gamma\left(\gamma_{1}\right)} \int_{0}^{s}(s-\tau)^{\gamma_{1}-1}|u(\tau)-v(\tau)| d \tau\right) d s \\
& +\frac{1}{\Gamma(\alpha)} \int_{t_{0}}^{1}(1-s)^{\alpha-1} \mu_{2}(s)(|u(s)-v(s)| \\
& +\frac{1}{\Gamma\left(1-\beta_{2}\right)} \int_{0}^{s}(s-\tau)^{-\beta_{2}}\left|u^{\prime}(\tau)-v^{\prime}(\tau)\right| d \tau \\
& \left.+\frac{1}{\Gamma\left(\gamma_{2}\right)} \int_{0}^{s}(s-\tau)^{\gamma_{2}-1}|u(\tau)-v(\tau)| d \tau\right) d s \\
& \leq \frac{1}{\Gamma(\alpha)} \int_{0}^{t}(t-s)^{\alpha-1} \mu_{1}(s) \\
& \times\left(1+\frac{1}{\Gamma\left(2-\beta_{1}\right)}+\frac{1}{\Gamma\left(1+\gamma_{1}\right)}\right)\|u-v\|_{*} d s \\
& +2 v_{1}\left(t_{0}\right)\left(1+\frac{1}{\Gamma\left(2-\beta_{3}\right)}+\frac{1}{\Gamma\left(1+\gamma_{3}\right)}\right)\|u-v\|_{*} \\
& +v_{2}\left(t_{0}\right)\left(1+\frac{1}{\Gamma\left(2-\beta_{4}\right)}+\frac{1}{\Gamma\left(1+\gamma_{4}\right)}\right)\|u-v\|_{*} \\
& +\frac{1}{\Gamma(\alpha)} \int_{0}^{t_{0}}(1-s)^{\alpha-1} \mu_{1}(s) \\
& \times\left(1+\frac{1}{\Gamma\left(2-\beta_{1}\right)}+\frac{1}{\Gamma\left(1+\gamma_{1}\right)}\right)\|u-v\|_{*} d s \\
& +\frac{1}{\Gamma(\alpha)} \int_{t_{0}}^{1}(1-s)^{\alpha-1} \mu_{2}(s) \\
& \times\left(1+\frac{1}{\Gamma\left(2-\beta_{2}\right)}+\frac{1}{\Gamma\left(1+\gamma_{2}\right)}\right)\|u-v\|_{*} d s \\
& \leq \frac{\|u-v\|_{*}}{\Gamma(\alpha)}\left(1+\frac{1}{\Gamma\left(2-\beta_{1}\right)}+\frac{1}{\Gamma\left(1+\gamma_{1}\right)}\right) \\
& \times\left[\int_{0}^{t}\left((t-s)^{\alpha-1}\right)^{\frac{1}{1-k}} d s\right]^{1-k}\left[\int_{0}^{t}\left(\mu_{1}(s)\right)^{\frac{1}{k}} d s\right]^{k} \\
& +\left[2\left\|v_{1}\right\|\left(1+\frac{1}{\Gamma\left(2-\beta_{3}\right)}+\frac{1}{\Gamma\left(1+\gamma_{3}\right)}\right)\right. \\
& \left.+\left\|v_{2}\right\|\left(1+\frac{1}{\Gamma\left(2-\beta_{4}\right)}+\frac{1}{\Gamma\left(1+\gamma_{4}\right)}\right)\right]\|u-v\|_{*} \\
& +\frac{\|u-v\|_{*}}{\Gamma(\alpha)}\left(1+\frac{1}{\Gamma\left(2-\beta_{1}\right)}+\frac{1}{\Gamma\left(1+\gamma_{1}\right)}\right)
\end{aligned}
$$




$$
\begin{aligned}
& \times\left[\int_{0}^{t_{0}}\left((1-s)^{\alpha-1}\right)^{\frac{1}{1-k}} d s\right]^{1-k}\left[\int_{0}^{t_{0}}\left(\mu_{1}(s)\right)^{\frac{1}{k}} d s\right]^{k} \\
& +\frac{\|u-v\|_{*}}{\Gamma(\alpha)}\left(1+\frac{1}{\Gamma\left(2-\beta_{2}\right)}+\frac{1}{\Gamma\left(1+\gamma_{2}\right)}\right) \\
& \times\left[\int_{t_{0}}^{1}\left((1-s)^{\alpha-1}\right)^{\frac{1}{1-k}} d s\right]^{1-k}\left[\int_{t_{0}}^{1}\left(\mu_{2}(s)\right)^{\frac{1}{k}} d s\right]^{k} \\
& \leq\left[\frac{2\left\|\mu_{1}\right\|_{\frac{1}{k}}}{\Gamma(\alpha)}\left(1+\frac{1}{\Gamma\left(2-\beta_{1}\right)}+\frac{1}{\Gamma\left(1+\gamma_{1}\right)}\right)\left(\frac{1-k}{\alpha-k}\right)^{1-k}\right. \\
& +\frac{\left\|\mu_{2}\right\|_{\frac{1}{k}}}{\Gamma(\alpha)}\left(1+\frac{1}{\Gamma\left(2-\beta_{2}\right)}+\frac{1}{\Gamma\left(1+\gamma_{2}\right)}\right)\left(\frac{1-k}{\alpha-k}\right)^{1-k} \\
& +2\left\|v_{1}\right\|\left(1+\frac{1}{\Gamma\left(2-\beta_{3}\right)}+\frac{1}{\Gamma\left(1+\gamma_{3}\right)}\right) \\
& \left.+\left\|v_{2}\right\|\left(1+\frac{1}{\Gamma\left(2-\beta_{4}\right)}+\frac{1}{\Gamma\left(1+\gamma_{4}\right)}\right)\right]\|u-v\|_{*}
\end{aligned}
$$

and

$$
\begin{aligned}
& \left|(N u)^{\prime}(t)-(N v)^{\prime}(t)\right|=\mid \frac{1}{\Gamma(\alpha-1)} \int_{0}^{t}(t-s)^{\alpha-2} \\
& \times f\left(s, u(s),{ }^{c} D^{\beta_{1}} u(s), I^{\gamma_{1}} u(s)\right) d s+\Delta_{u}\left(t_{0}\right) \\
& -\frac{1}{\Gamma(\alpha-1)} \int_{0}^{t}(t-s)^{\alpha-2} \\
& \times f_{1}\left(s, v(s),{ }^{c} D^{\beta_{1}} v(s), I^{\gamma_{1}} v(s)\right) d s-\Delta_{v}\left(t_{0}\right) \\
& \leq\left[\frac{\left\|\mu_{1}\right\|_{\frac{1}{k}}}{\Gamma(\alpha-1)}\left(1+\frac{1}{\Gamma\left(2-\beta_{1}\right)}+\frac{1}{\Gamma\left(1+\gamma_{1}\right)}\right)\right. \\
& \times\left(\frac{1-k}{\alpha-k+1}\right)^{1-k} \\
& +\frac{\left\|\mu_{1}\right\|_{\frac{1}{k}}}{\Gamma(\alpha)}\left(1+\frac{1}{\Gamma\left(2-\beta_{1}\right)}+\frac{1}{\Gamma\left(1+\gamma_{1}\right)}\right) \\
& \times\left(\frac{1-k}{\alpha-k}\right)^{1-k} \\
& +\frac{\left\|\mu_{2}\right\|_{\frac{1}{k}}}{\Gamma(\alpha)}\left(1+\frac{1}{\Gamma\left(2-\beta_{2}\right)}+\frac{1}{\Gamma\left(1+\gamma_{2}\right)}\right) \\
& \times\left(\frac{1-k}{\alpha-k}\right)^{1-k} \\
& +\left\|v_{1}\right\|\left(1+\frac{1}{\Gamma\left(2-\beta_{3}\right)}+\frac{1}{\Gamma\left(1+\gamma_{3}\right)}\right) \\
& \left.+\left\|v_{2}\right\|\left(1+\frac{1}{\Gamma\left(2-\beta_{4}\right)}+\frac{1}{\Gamma\left(1+\gamma_{4}\right)}\right)\right]\|u-v\|_{*} \text {. }
\end{aligned}
$$


If $t_{0} \leq t \leq 1$, then we have

$$
\begin{aligned}
& |N u(t)-N v(t)|=\mid \frac{1}{\Gamma(\alpha)} \int_{0}^{t_{0}}(t-s)^{\alpha-1} f_{1}\left(s, u(s),{ }^{c} D^{\beta_{1}} u(s), I^{\gamma_{1}} u(s)\right) d s \\
& +\frac{1}{\Gamma(\alpha)} \int_{t_{0}}^{t}(t-s)^{\alpha-1} f_{2}\left(s, u(s),{ }^{c} D^{\beta_{2}} u(s), I^{\gamma_{2}} f_{2}(s)\right) d s \\
& +h_{1}\left(t_{0}, u\left(t_{0}\right),{ }^{c} D^{\beta_{3}} u\left(t_{0}\right), I^{\gamma_{3}} u\left(t_{0}\right)\right)+\Delta_{u}\left(t_{0}\right) t \\
& -\frac{1}{\Gamma(\alpha)} \int_{0}^{t_{0}}(t-s)^{\alpha-1} f\left(s, v(s),{ }^{c} D^{\beta_{1}} v(s), I^{\gamma_{1}} v(s)\right) d s \\
& -\frac{1}{\Gamma(\alpha)} \int_{t_{0}}^{t}(t-s)^{\alpha-1} f_{2}\left(s, v(s),{ }^{c} D^{\beta_{2}} v(s), I^{\gamma_{2}} v(s)\right) d s \\
& -h_{1}\left(t_{0}, v\left(t_{0}\right),{ }^{c} D^{\beta_{3}} v\left(t_{0}\right), I^{\gamma_{3}} v\left(t_{0}\right)\right)-\Delta_{v}\left(t_{0}\right) t \\
& \leq\left[\frac{2\left\|\mu_{1}\right\|_{\frac{1}{k}}}{\Gamma(\alpha)}\left(1+\frac{1}{\Gamma\left(2-\beta_{1}\right)}+\frac{1}{\Gamma\left(1+\gamma_{1}\right)}\right)\left(\frac{1-k}{\alpha-k}\right)^{1-k}\right. \\
& +\frac{2\left\|\mu_{2}\right\|_{\frac{1}{k}}}{\Gamma(\alpha)}\left(1+\frac{1}{\Gamma\left(2-\beta_{2}\right)}+\frac{1}{\Gamma\left(1+\gamma_{2}\right)}\right)\left(\frac{1-k}{\alpha-k}\right)^{1-k} \\
& +2\left\|v_{1}\right\|\left(1+\frac{1}{\Gamma\left(2-\beta_{3}\right)}+\frac{1}{\Gamma\left(1+\gamma_{3}\right)}\right) \\
& \left.+\left\|v_{2}\right\|\left(1+\frac{1}{\Gamma\left(2-\beta_{4}\right)}+\frac{1}{\Gamma\left(1+\gamma_{4}\right)}\right)\right]\|u-v\|_{*}
\end{aligned}
$$

and

$$
\begin{aligned}
\left|(N u)^{\prime}(t)-(N v)^{\prime}(t)\right|=\mid & \frac{1}{\Gamma(\alpha-1)} \int_{0}^{t_{0}}(t-s)^{\alpha-2} \\
& \times f_{1}\left(s, u(s),{ }^{c} D^{\beta_{1}} u(s), I^{\gamma_{1}} u(s)\right) d s \\
& +\frac{1}{\Gamma(\alpha-1)} \int_{t_{0}}^{t}(t-s)^{\alpha-2} \\
& \times f_{2}\left(s, u(s),{ }^{c} D^{\beta_{2}} x(s), I^{\gamma_{2}} u(s)\right) d s \\
& +\Delta_{u}\left(t_{0}\right)-h_{1}\left(t_{0}, u\left(t_{0}\right),{ }^{c} D^{\beta_{3}} u\left(t_{0}\right), I^{\gamma_{3}} u\left(t_{0}\right)\right) \\
& -\frac{1}{\Gamma(\alpha-1)} \int_{0}^{t_{0}}(t-s)^{\alpha-2} \\
& \times f_{1}\left(s, v(s),{ }^{c} D^{\beta_{1}} v(s), I^{\gamma_{1}} v(s)\right) d s \\
& -\frac{1}{\Gamma(\alpha-1)} \int_{t_{0}}^{t}(t-s)^{\alpha-2} \\
& \times f_{2}\left(s, v(s),{ }^{c} D^{\beta_{2}} v(s), I^{\gamma_{2}} v(s)\right) d s-\Delta_{v}\left(t_{0}\right) \mid \\
\leq & \left.\times \frac{\left\|\mu_{1}\right\| \frac{1}{k}}{\Gamma(\alpha-1)}\left(1+\frac{1-k}{\alpha-k+1}\right)^{1-k} \frac{1}{\Gamma\left(2-\beta_{1}\right)}+\frac{1}{\Gamma\left(1+\gamma_{1}\right)}\right) \\
& \times\left(\frac{1}{\alpha(\alpha)}\right) \\
& \\
& \\
&
\end{aligned}
$$




$$
\begin{aligned}
& +\frac{\left\|\mu_{2}\right\|_{\frac{1}{k}}}{\Gamma(\alpha-1)}\left(1+\frac{1}{\Gamma\left(2-\beta_{2}\right)}+\frac{1}{\Gamma\left(1+\gamma_{2}\right)}\right) \\
& \times\left(\frac{1-k}{\alpha-k+1}\right)^{1-k} \\
& +\frac{\left\|\mu_{1}\right\|_{\frac{1}{k}}}{\Gamma(\alpha)}\left(1+\frac{1}{\Gamma\left(2-\beta_{1}\right)}+\frac{1}{\Gamma\left(1+\gamma_{1}\right)}\right) \\
& \times\left(\frac{1-k}{\alpha-k}\right)^{1-k} \\
& +\frac{\left\|\mu_{2}\right\|_{\frac{1}{k}}}{\Gamma(\alpha)}\left(1+\frac{1}{\Gamma\left(2-\beta_{2}\right)}+\frac{1}{\Gamma\left(1+\gamma_{2}\right)}\right) \\
& \times\left(\frac{1-k}{\alpha-k}\right)^{1-k} \\
& +\left\|v_{1}\right\|\left(1+\frac{1}{\Gamma\left(2-\beta_{3}\right)}+\frac{1}{\Gamma\left(1+\gamma_{3}\right)}\right) \\
& \left.+\left\|v_{2}\right\|\left(1+\frac{1}{\Gamma\left(2-\beta_{4}\right)}+\frac{1}{\Gamma\left(1+\gamma_{4}\right)}\right)\right]\|u-v\|_{*} .
\end{aligned}
$$

By (3), (4), (5), and (6), we have

$$
\begin{aligned}
\|N u-N v\|_{*}= & \|N u-N v\|+\left\|(N u)^{\prime}-(N v)^{\prime}\right\| \\
\leq & {\left[\frac{3\left\|\mu_{1}\right\|_{\frac{1}{k}}}{\Gamma(\alpha)}\left(1+\frac{1}{\Gamma\left(2-\beta_{1}\right)}+\frac{1}{\Gamma\left(1+\gamma_{1}\right)}\right)\left(\frac{1-k}{\alpha-k}\right)^{1-k}\right.} \\
& +\frac{3\left\|\mu_{2}\right\|_{\frac{1}{k}}}{\Gamma(\alpha)}\left(1+\frac{1}{\Gamma\left(2-\beta_{2}\right)}+\frac{1}{\Gamma\left(1+\gamma_{2}\right)}\right)\left(\frac{1-k}{\alpha-k}\right)^{1-k} \\
& +3\left\|v_{1}\right\|\left(1+\frac{1}{\Gamma\left(2-\beta_{3}\right)}+\frac{1}{\Gamma\left(1+\gamma_{3}\right)}\right) \\
& +2\left\|v_{2}\right\|\left(1+\frac{1}{\Gamma\left(2-\beta_{4}\right)}+\frac{1}{\Gamma\left(1+\gamma_{4}\right)}\right) \\
& +\frac{\left\|\mu_{1}\right\|_{\frac{1}{k}}}{\Gamma(\alpha-1)}\left(1+\frac{1}{\Gamma\left(2-\beta_{1}\right)}+\frac{1}{\Gamma\left(1+\gamma_{1}\right)}\right)\left(\frac{1-k}{\alpha-k+1}\right)^{1-k} \\
& \left.+\frac{\left\|\mu_{2}\right\|_{\frac{1}{k}}}{\Gamma(\alpha-1)}\left(1+\frac{1}{\Gamma\left(2-\beta_{2}\right)}+\frac{1}{\Gamma\left(1+\gamma_{2}\right)}\right)\left(\frac{1-k}{\alpha-k+1}\right)^{1-k}\right] \\
& \times\|u-v\|_{*} \\
= & \Lambda\|u-v\|_{*} .
\end{aligned}
$$

Thus $N$ is a contraction mapping, because $\Lambda<1$. Therefore, $N$ satisfies the Banach contraction principle, and so does a unique fixed point which is the unique solution of problem (1) by applying Corollary 1. 
Corollary 2 Problem (1) has a unique solution whenever there exist $l_{1}, l_{2}, l_{3}$, and $l_{4} \in \mathbb{R}^{+}$ such that

$$
\begin{aligned}
& \left|f_{1}\left(t, x_{1}, x_{2}, x_{3}\right)-f_{1}\left(t, x_{1}^{\prime}, x_{2}^{\prime}, x_{3}^{\prime}\right)\right| \leq l_{1} \sum_{j=1}^{3}\left|x_{j}-x_{j}^{\prime}\right|, \\
& \left|f_{2}\left(t, x_{1}, x_{2}, x_{3}\right)-f_{2}\left(t, x_{1}^{\prime}, x_{2}^{\prime}, x_{3}^{\prime}\right)\right| \leq l_{2} \sum_{j=1}^{3}\left|x_{j}-x_{j}^{\prime}\right|, \\
& \left|h_{1}\left(t, x_{1}, x_{2}, x_{3}\right)-h_{1}\left(t, x_{1}^{\prime}, x_{2}^{\prime}, x_{3}^{\prime}\right)\right| \leq l_{3} \sum_{j=1}^{3}\left|x_{j}-x_{j}^{\prime}\right|, \\
& \left|h_{2}\left(t, x_{1}, x_{2}, x_{3}\right)-h_{2}\left(t, x_{1}^{\prime}, x_{2}^{\prime}, x_{3}^{\prime}\right)\right| \leq l_{4} \sum_{j=1}^{3}\left|x_{j}-x_{j}^{\prime}\right|,
\end{aligned}
$$

and

$$
\begin{aligned}
& \frac{3 L_{1}}{\Gamma(\alpha+1)}\left(1+\frac{1}{\Gamma\left(2-\beta_{1}\right)}+\frac{1}{\Gamma\left(1+\gamma_{1}\right)}\right) \\
& +\frac{3 L_{2}}{\Gamma(\alpha+1)}\left(1+\frac{1}{\Gamma\left(2-\beta_{2}\right)}+\frac{1}{\Gamma\left(1+\gamma_{2}\right)}\right) \\
& +3 L_{3}\left(1+\frac{1}{\Gamma\left(2-\beta_{3}\right)}+\frac{1}{\Gamma\left(1+\gamma_{3}\right)}\right) \\
& +2 L_{4}\left(1+\frac{1}{\Gamma\left(2-\beta_{4}\right)}+\frac{1}{\Gamma\left(1+\gamma_{4}\right)}\right) \\
& +\frac{L_{1}}{\Gamma(\alpha)}\left(1+\frac{1}{\Gamma\left(2-\beta_{1}\right)}+\frac{1}{\Gamma\left(1+\gamma_{1}\right)}\right) \\
& +\frac{L_{2}}{\Gamma(\alpha)}\left(1+\frac{1}{\Gamma\left(2-\beta_{2}\right)}+\frac{1}{\Gamma\left(1+\gamma_{2}\right)}\right)<1
\end{aligned}
$$

for each $t \in J$ and $x_{j}, x_{j}^{\prime} \in \mathbb{R}$.

Our next existence result is based on Krasnoselskii's fixed point theorem.

Theorem 10 Equation (1) has at least one solution on $[0,1]$, whenever there exist $\mu_{i}, v_{i} \in$ $C(\bar{J},[0, \infty))$ and nondecreasing functions $\psi_{i}: \mathbb{R}^{+} \rightarrow \mathbb{R}^{+}$, for $i=1,2$, such that

$$
\begin{aligned}
& \left|f_{1}\left(t, x_{1}, x_{2}, x_{3}\right)-f_{1}\left(t, x_{1}^{\prime}, x_{2}^{\prime}, x_{3}^{\prime}\right)\right| \leq \mu_{1}(t) \sum_{j=1}^{3}\left|x_{j}-x_{j}^{\prime}\right|, \\
& \left|f_{2}\left(t, x_{1}, x_{2}, x_{3}\right)-f_{2}\left(t, x_{1}^{\prime}, x_{2}^{\prime}, x_{3}^{\prime}\right)\right| \leq \mu_{2}(t) \sum_{j=1}^{3}\left|x_{j}-x_{j}^{\prime}\right|, \\
& \left|h_{1}\left(t, x_{1}, x_{2}, x_{3}\right)\right| \leq v_{1}(t) \psi_{1}\left(\sum_{j=1}^{3}\left|x_{j}\right|\right), \\
& \left|h_{2}\left(t, x_{1}, x_{2}, x_{3}\right)\right| \leq v_{2}(t) \psi_{2}\left(\sum_{j=1}^{3}\left|x_{j}\right|\right),
\end{aligned}
$$


and

$$
\begin{aligned}
\Delta= & {\left[\frac{\left\|\mu_{1}\right\|}{\Gamma(\alpha)}\left(1+\frac{1}{\Gamma\left(2-\beta_{1}\right)}+\frac{1}{\Gamma\left(1+\gamma_{1}\right)}\right)\right.} \\
& \left.+\frac{\left\|\mu_{2}\right\|}{\Gamma(\alpha)}\left(1+\frac{1}{\Gamma\left(2-\beta_{2}\right)}+\frac{1}{\Gamma\left(1+\gamma_{2}\right)}\right)\right]\left(\frac{1}{\alpha}+1\right)
\end{aligned}
$$

$<1$,

for almost all $t \in \bar{J}$ and $x_{j}, x_{j}^{\prime} \in \mathbb{R}$.

Proof Consider the set of all $u \in \mathcal{X}$ somehow that $\|u\| \leq r$, and denote by $S$, where

$$
\begin{aligned}
& 3\left\|v_{1}\right\| \psi_{1}\left(\left(1+\frac{1}{\Gamma\left(2-\beta_{3}\right)}+\frac{1}{\Gamma\left(1+\gamma_{3}\right)}\right) r\right) \\
& +2\left\|v_{2}\right\| \psi_{2}\left(\left(1+\frac{1}{\Gamma\left(2-\beta_{4}\right)}+\frac{1}{\Gamma\left(1+\gamma_{4}\right)}\right) r\right) \\
& +\frac{r}{\Gamma(\alpha)}\left(\frac{2}{\alpha}+\alpha+1\right)\left[\left\|\mu_{1}\right\|\left(1+\frac{1}{\Gamma\left(2-\beta_{1}\right)}+\frac{1}{\Gamma\left(1+\gamma_{1}\right)}\right)+f_{1}^{0}\right] \\
& +\frac{r}{\Gamma(\alpha)}\left(\frac{2}{\alpha}+\alpha+1\right)\left[\left\|\mu_{2}\right\|\left(1+\frac{1}{\Gamma\left(2-\beta_{2}\right)}+\frac{1}{\Gamma\left(1+\gamma_{2}\right)}\right)+f_{2}^{0}\right]
\end{aligned}
$$$$
\leq r \text {. }
$$

Clearly $S$ is the closed convex and nonempty subset of a Banach space $\mathcal{X}$. We define the operators $A$ and $B$ on $S$ as

$$
A u(t)=h_{1}\left(t_{0}, u\left(t_{0}\right),{ }^{c} D^{\beta_{3}} u\left(t_{0}\right), I^{\gamma_{3}} u\left(t_{0}\right)\right)+\Delta_{u}\left(t_{0}\right) t
$$

for all $0 \leq t \leq 1$, and $B u(t)=\frac{1}{\Gamma(\alpha)} \int_{0}^{t}(t-s)^{\alpha-1} f_{1}\left(s, u(s),{ }^{c} D^{\beta_{1}} u(s), I^{\gamma_{1}} u(s)\right) d s$ whenever $0 \leq t \leq$ $t_{0}$,

$$
\begin{aligned}
B u(t)= & \frac{1}{\Gamma(\alpha)} \int_{0}^{t_{0}}(t-s)^{\alpha-1} f_{1}\left(s, u(s),{ }^{c} D^{\beta_{1}} u(s), I^{\gamma_{1}} u(s)\right) d s \\
& +\frac{1}{\Gamma(\alpha)} \int_{t_{0}}^{t}(t-s)^{\alpha-1} f_{2}\left(s, u(s),{ }^{c} D^{\beta_{2}} u(s), I^{\gamma_{2}} u(s)\right) d s
\end{aligned}
$$

whenever $t_{0} \leq t \leq 1$. Let $u, v \in S$. For each $0 \leq t \leq t_{0}$, we have

$$
\begin{aligned}
|A u(t)+B v(t)|= & \mid h_{1}\left(t_{0}, u\left(t_{0}\right),{ }^{c} D^{\beta_{3}} u\left(t_{0}\right), I^{\gamma_{3}} u\left(t_{0}\right)\right)+\Delta_{u}\left(t_{0}\right) t \\
& +\frac{1}{\Gamma(\alpha)} \int_{0}^{t}(t-s)^{\alpha-1} f_{1}\left(s, v(s),{ }^{c} D^{\beta_{1}} v(s), I^{\gamma_{1}} v(s)\right) d s \mid \\
\leq & 2 v_{1}\left(t_{0}\right) \psi_{1}\left(\left|u\left(t_{0}\right)\right|+\left|{ }^{c} D^{\beta_{3}} u\left(t_{0}\right)\right|+\left|I^{\gamma_{3}} u\left(t_{0}\right)\right|\right) \\
& +v_{2}\left(t_{0}\right) \psi_{2}\left(\left|u\left(t_{0}\right)\right|+\left|{ }^{c} D^{\beta_{4}} u\left(t_{0}\right)\right|+\left|I^{\gamma_{4}} u\left(t_{0}\right)\right|\right) \\
& +\frac{1}{\Gamma(\alpha)} \int_{0}^{t_{0}}(1-s)^{\alpha-1}
\end{aligned}
$$




$$
\begin{aligned}
& \quad \times\left(\mu_{1}(s)\left|u(s)+{ }^{c} D^{\beta_{1}} u(s)+I^{\gamma_{1}} u(s)\right|+f_{1}^{0}\right) d s \\
& +\frac{1}{\Gamma(\alpha)} \int_{t_{0}}^{1}(1-s)^{\alpha-1} \\
& \times\left(\mu_{2}(s)\left|u(s)+{ }^{c} D^{\beta_{2}} u(s)+I^{\gamma_{2}} u(s)\right|+f_{2}^{0}\right) d s \\
& +\frac{1}{\Gamma(\alpha)} \int_{0}^{t}(t-s)^{\alpha-1} \\
& \times\left(\mu_{1}(s)\left|\mu(s)+{ }^{c} D^{\beta_{1}} v(s)+I^{\gamma_{1}} v(s)\right|+f_{1}^{0}\right) d s \\
& \leq 2\left\|v_{1}\right\| \psi_{1}\left(\left(1+\frac{1}{\Gamma\left(2-\beta_{3}\right)}+\frac{1}{\Gamma\left(1+\gamma_{3}\right)}\right) r\right) \\
& +\left\|v_{2}\right\| \psi_{2}\left(\left(1+\frac{1}{\Gamma\left(2-\beta_{4}\right)}+\frac{1}{\Gamma\left(1+\gamma_{4}\right)}\right) r\right) \\
& +\frac{r}{\Gamma(\alpha+1)}\left[2\left\|L_{1}\right\|\left(1+\frac{1}{\Gamma\left(2-\beta_{1}\right)}+\frac{1}{\Gamma\left(1+\gamma_{1}\right)}\right)+2 f_{1}^{0}\right. \\
& \left.+\left\|\mu_{2}\right\|\left(1+\frac{1}{\Gamma\left(2-\beta_{2}\right)}+\frac{1}{\Gamma\left(1+\gamma_{2}\right)}\right)+f_{2}^{0}\right]
\end{aligned}
$$

and

$$
\begin{aligned}
\left|(A u)^{\prime}(t)+(B v)^{\prime}(t)\right|= & h_{2}\left(t_{0}, u\left(t_{0}\right),{ }^{c} D^{\beta_{4}} u\left(t_{0}\right), I^{\gamma_{4}} u\left(t_{0}\right)\right) \\
& -\frac{1}{\Gamma(\alpha)} \int_{0}^{t_{0}}(1-s)^{\alpha-1} f_{1}\left(s, u(s),{ }^{c} D^{\beta_{1}} u(s), I^{\gamma_{1}} u(s)\right) d s \\
& -\frac{1}{\Gamma(\alpha)} \int_{t_{0}}^{1}(1-s)^{\alpha-1} f_{2}\left(s, u(s),{ }^{c} D^{\beta_{2}} u(s), I^{\gamma_{2}} u(s)\right) d s \\
& -h_{1}\left(t_{0}, u\left(t_{0}\right),{ }^{c} D^{\beta_{3}} u\left(t_{0}\right), I^{\gamma_{3}} u\left(t_{0}\right)\right) \\
& +\frac{1}{\Gamma(\alpha-1)} \int_{0}^{t}(t-s)^{\alpha-2} \\
& \times f_{1}\left(s, v(s),{ }^{c} D^{\beta_{1}} v(s), I^{\gamma_{1}} v(s)\right) d s \mid \\
\leq & \left\|v_{2}\right\| \psi_{2}\left(\left(1+\frac{1}{\Gamma\left(2-\beta_{4}\right)}+\frac{1}{\Gamma\left(1+\gamma_{4}\right)}\right) r\right) \\
& +\left\|v_{1}\right\| \psi_{1}\left(\left(1+\frac{1}{\Gamma\left(2-\beta_{3}\right)}+\frac{1}{\Gamma\left(1+\gamma_{3}\right)}\right) r\right) \\
& +\frac{r(\alpha+1)}{\Gamma(\alpha)}\left[\left\|\mu_{1}\right\|\left(1+\frac{1}{\Gamma\left(2-\beta_{1}\right)}+\frac{1}{\Gamma\left(1+\gamma_{1}\right)}\right)+f_{1}^{0}\right] \\
& +\frac{r}{\Gamma(\alpha+1)}\left[\left\|\mu_{2}\right\|\left(1+\frac{1}{\Gamma\left(2-\beta_{2}\right)}+\frac{1}{\Gamma\left(1+\gamma_{2}\right)}\right)+f_{2}^{0}\right] .
\end{aligned}
$$

Also, if $t_{0} \leq t \leq 1$, we have

$$
\begin{aligned}
|A u(t)+B v(t)| \leq & 2 v_{1}\left(t_{0}\right) \psi_{1}\left(\left|u\left(t_{0}\right)\right|+\left|{ }^{c} D^{\beta_{3}} u\left(t_{0}\right)\right|+\left|I^{\gamma_{3}} u\left(t_{0}\right)\right|\right) \\
& +v_{2}\left(t_{0}\right) \psi_{2}\left(\left|u\left(t_{0}\right)\right|+\left|{ }^{c} D^{\beta_{4}} u\left(t_{0}\right)\right|+\left|I^{\gamma_{4}} u\left(t_{0}\right)\right|\right)
\end{aligned}
$$


Hedayati and Samei Boundary Value Problems

(2019) 2019:141

Page 16 of 23

$$
\begin{aligned}
& +\frac{1}{\Gamma(\alpha)} \int_{0}^{t_{0}}(1-s)^{\alpha-1} \\
& \times\left(\mu_{1}(s)\left|u(s)+{ }^{c} D^{\beta_{1}} u(s)+I^{\gamma_{1}} u(s)\right|+f_{1}^{0}\right) d s \\
& +\frac{1}{\Gamma(\alpha)} \int_{t_{0}}^{1}(1-s)^{\alpha-1} \\
& \times\left(\mu_{2}(s)\left|u(s)+{ }^{c} D^{\beta_{2}} u(s)+I^{\gamma_{2}} u(s)\right|+f_{2}^{0}\right) d s \\
& +\frac{1}{\Gamma(\alpha)} \int_{0}^{t_{0}}(t-s)^{\alpha-1} \\
& \times\left(\mu_{1}(s)\left|v(s)+{ }^{c} D^{\beta_{1}} v(s)+I^{\gamma_{1}} v(s)\right|+f_{1}^{0}\right) d s \\
& +\frac{1}{\Gamma(\alpha)} \int_{t_{0}}^{t}(t-s)^{\alpha-1} \\
& \times\left(\mu_{2}(s)\left|v(s)+{ }^{c} D^{\beta_{2}} v(s)+I^{\gamma_{2}} v(s)\right|+f_{2}^{0}\right) d s \\
& \leq 2\left\|v_{1}\right\| \psi_{1}\left(\left(1+\frac{1}{\Gamma\left(2-\beta_{3}\right)}+\frac{1}{\Gamma\left(1+\gamma_{3}\right)}\right) r\right) \\
& \left.+\left\|\mu_{2}\right\|\left(1+\frac{1}{\Gamma\left(2-\beta_{2}\right)}+\frac{1}{\Gamma\left(1+\gamma_{2}\right)}\right)+f_{2}^{0}\right] \\
& +\|(\alpha+1)
\end{aligned}
$$

and

$$
\begin{aligned}
\left|(A u)^{\prime}(t)+(B v)^{\prime}(t)\right|= & \mid \Delta_{u}\left(t_{0}\right)+\frac{1}{\Gamma(\alpha-1)} \int_{0}^{t_{0}}(t-s)^{\alpha-2} \\
& \times f_{1}\left(s, v(s),{ }^{c} D^{\beta_{1}} v(s), I^{\gamma_{1}} v(s)\right) d s \\
& +\frac{1}{\Gamma(\alpha-1)} \int_{t_{0}}^{t}(t-s)^{\alpha-2} \\
& \times f_{2}\left(s, v(s),{ }^{c} D^{\beta_{2}} v(s), I^{\gamma_{2}} v(s)\right) d s \mid \\
\leq & \left\|v_{2}\right\| \psi_{2}\left(\left(1+\frac{1}{\Gamma\left(2-\beta_{4}\right)}+\frac{1}{\Gamma\left(1+\gamma_{4}\right)}\right) r\right) \\
& +\left\|v_{1}\right\| \psi_{1}\left(\left(1+\frac{1}{\Gamma\left(2-\beta_{3}\right)}+\frac{1}{\Gamma\left(1+\gamma_{3}\right)}\right) r\right) \\
& +\frac{r(\alpha+1)}{\Gamma(\alpha)} \\
& \times\left[\left\|\mu_{1}\right\|\left(1+\frac{1}{\Gamma\left(2-\beta_{1}\right)}+\frac{1}{\Gamma\left(1+\gamma_{1}\right)}\right)+f_{1}^{0}\right] \\
& +\frac{r(\alpha+1)}{\Gamma(\alpha)} \\
& \times\left[\left\|\mu_{2}\right\|\left(1+\frac{1}{\Gamma\left(2-\beta_{2}\right)}+\frac{1}{\Gamma\left(1+\gamma_{2}\right)}\right)+f_{2}^{0}\right],
\end{aligned}
$$


where $f_{i}^{0}=\sup _{t \in \bar{J}}\left|f_{i}(t, 0,0,0)\right|$ for $i=1,2$. Thus

$$
\begin{aligned}
\|A u+B v\|_{*}= & \|A u+B v\|+\left\|(A u)^{\prime}+(B v)^{\prime}\right\| \\
\leq & 2\left\|v_{1}\right\| \psi_{1}\left(\left(1+\frac{1}{\Gamma\left(2-\beta_{3}\right)}+\frac{1}{\Gamma\left(1+\gamma_{3}\right)}\right) r\right) \\
& +\left\|v_{2}\right\| \psi_{2}\left(\left(1+\frac{1}{\Gamma\left(2-\beta_{4}\right)}+\frac{1}{\Gamma\left(1+\gamma_{4}\right)}\right) r\right) \\
& +\frac{2 r}{\Gamma(\alpha+1)}\left[\left\|\mu_{1}\right\|\left(1+\frac{1}{\Gamma\left(2-\beta_{1}\right)}+\frac{1}{\Gamma\left(1+\gamma_{1}\right)}\right)+f_{1}^{0}\right. \\
& \left.+\left\|\mu_{2}\right\|\left(1+\frac{1}{\Gamma\left(2-\beta_{2}\right)}+\frac{1}{\Gamma\left(1+\gamma_{2}\right)}\right)+f_{2}^{0}\right] \\
& +\left\|v_{2}\right\| \psi_{2}\left(\left(1+\frac{1}{\Gamma\left(2-\beta_{4}\right)}+\frac{1}{\Gamma\left(1+\gamma_{4}\right)}\right) r\right) \\
& +\left\|v_{1}\right\| \psi_{1}\left(\left(1+\frac{1}{\Gamma\left(2-\beta_{3}\right)}+\frac{1}{\Gamma\left(1+\gamma_{3}\right)}\right) r\right) \\
& +\frac{r(\alpha+1)}{\Gamma(\alpha)}\left[\left\|\mu_{1}\right\|\left(1+\frac{1}{\Gamma\left(2-\beta_{1}\right)}+\frac{1}{\Gamma\left(1+\gamma_{1}\right)}\right)+f_{1}^{0}\right] \\
& +\frac{r(\alpha+1)}{\Gamma(\alpha)}\left[\left\|\mu_{2}\right\|\left(1+\frac{1}{\Gamma\left(2-\beta_{2}\right)}+\frac{1}{\Gamma\left(1+\gamma_{2}\right)}\right)+f_{2}^{0}\right] \\
= & 3\left\|v_{1}\right\| \psi_{1}\left(\left(1+\frac{1}{\Gamma\left(2-\beta_{3}\right)}+\frac{1}{\Gamma\left(1+\gamma_{3}\right)}\right) r\right) \\
& +2\left\|v_{2}\right\| \psi_{2}\left(\left(1+\frac{1}{\Gamma\left(2-\beta_{4}\right)}+\frac{1}{\Gamma\left(1+\gamma_{4}\right)}\right) r\right) \\
& +\frac{r}{\Gamma(\alpha)}\left(\frac{2}{\alpha}+\alpha+1\right) \\
& \times\left[\left\|\mu_{1}\right\|\left(1+\frac{1}{\Gamma\left(2-\beta_{1}\right)}+\frac{1}{\Gamma\left(1+\gamma_{1}\right)}\right)+f_{1}^{0}\right] \\
& {\left[\left\|\mu_{2}\right\|\left(1+\frac{1}{\Gamma\left(2-\beta_{2}\right)}+\frac{1}{\Gamma\left(1+\gamma_{2}\right)}\right)+f_{2}^{0}\right] } \\
& {[1) }
\end{aligned}
$$

Hence, for each $u, v \in S, A u+B v \in S$. On the other hand, for each $u \in S$, we get

$$
\begin{aligned}
\|A u\|_{*} \leq & 3\left\|\nu_{1}\right\| \psi_{1}\left(\left(1+\frac{1}{\Gamma\left(2-\beta_{3}\right)}+\frac{1}{\Gamma\left(1+\gamma_{3}\right)}\right) r\right) \\
& +2\left\|v_{2}\right\| \psi_{2}\left(\left(1+\frac{1}{\Gamma\left(2-\beta_{4}\right)}+\frac{1}{\Gamma\left(1+\gamma_{4}\right)}\right) r\right) \\
& +\frac{2 r}{\Gamma(\alpha+1)}\left[\left\|\mu_{1}\right\|\left(1+\frac{1}{\Gamma\left(2-\beta_{1}\right)}+\frac{1}{\Gamma\left(1+\gamma_{1}\right)}\right)+f_{1}^{0}\right. \\
& \left.+\left\|\mu_{2}\right\|\left(1+\frac{1}{\Gamma\left(2-\beta_{2}\right)}+\frac{1}{\Gamma\left(1+\gamma_{2}\right)}\right)+f_{2}^{0}\right] .
\end{aligned}
$$


Thus, $A$ is uniformly bounded on $S$. Also, for any $u \in S$ and $t<\tau \in \bar{J}$, we have $\mid A u(\tau)-$ $A u(t) \mid=\Delta_{u}\left(t_{0}\right)(\tau-t)$, which is independent of $u$ and tends to zero as $t \rightarrow \tau$. Thus, $A$ is equicontinuous. Hence, by the Arzelá-Ascoli theorem, $A$ is compact on $S$. Now, we show that $B$ is a contraction map. Let $u, v \in S$. If $0 \leq t \leq t_{0}$, then we have

$$
\begin{aligned}
|B u(t)-B v(t)|= & \mid \frac{1}{\Gamma(\alpha)} \int_{0}^{t}(t-s)^{\alpha-1} f_{1}\left(s, u(s),{ }^{c} D^{\beta_{1}} u(s), I^{\gamma_{1}} u(s)\right) d s \\
& -\frac{1}{\Gamma(\alpha)} \int_{0}^{t}(t-s)^{\alpha-1} f_{1}\left(s, v(s),{ }^{c} D^{\beta_{1}} v(s), I^{\gamma_{1}} v(s)\right) d s \mid \\
\leq & \frac{\left\|\mu_{1}\right\|}{\Gamma(\alpha+1)}\left(1+\frac{1}{\Gamma\left(2-\beta_{1}\right)}+\frac{1}{\Gamma\left(1+\gamma_{1}\right)}\right)\|u-v\|_{*}
\end{aligned}
$$

and

$$
\begin{aligned}
\left|(B u)^{\prime}(t)-(B v)^{\prime}(t)\right|= & \mid \frac{1}{\Gamma(\alpha-1)} \int_{0}^{t}(t-s)^{\alpha-2} \\
& \times f_{1}\left(s, u(s),{ }^{c} D^{\beta_{1}} u(s), I^{\gamma_{1}} u(s)\right) d s \\
& -\frac{1}{\Gamma(\alpha-1)} \int_{0}^{t}(t-s)^{\alpha-2} \\
& \times f_{1}\left(s, v(s),{ }^{c} D^{\beta_{1}} v(s), I^{\gamma_{1}} v(s)\right) d s \mid \\
\leq & \frac{\left\|\mu_{1}\right\|}{\Gamma(\alpha)}\left(1+\frac{1}{\Gamma\left(2-\beta_{1}\right)}+\frac{1}{\Gamma\left(1+\gamma_{1}\right)}\right)\|u-v\|_{*} .
\end{aligned}
$$

Also, for $t_{0} \leq t \leq 1$, we obtain

$$
\begin{aligned}
|B u(t)-B v(t)|= & \mid \frac{1}{\Gamma(\alpha)} \int_{0}^{t_{0}}(t-s)^{\alpha-1} f_{1}\left(s, u(s),{ }^{c} D^{\beta_{1}} u(s), I^{\gamma_{1}} u(s)\right) d s \\
& +\frac{1}{\Gamma(\alpha)} \int_{t_{0}}^{t}(t-s)^{\alpha-1} f_{2}\left(s, u(s),{ }^{c} D^{\beta_{2}} u(s), I^{\gamma_{2}} x(s)\right) d s \\
& -\frac{1}{\Gamma(\alpha)} \int_{0}^{t_{0}}(t-s)^{\alpha-1} f_{1}\left(s, v(s),{ }^{c} D^{\beta_{1}} v(s), I^{\gamma_{1}} v(s)\right) d s \\
& -\frac{1}{\Gamma(\alpha)} \int_{t_{0}}^{t}(t-s)^{\alpha-1} f_{2}\left(s, v(s),{ }^{c} D^{\beta_{2}} v(s), I^{\gamma_{2}} v(s)\right) d s \mid \\
\leq & \|u-v\|_{*}\left[\frac{\left\|\mu_{1}\right\|}{\Gamma(\alpha+1)}\left(1+\frac{1}{\Gamma\left(2-\beta_{1}\right)}+\frac{1}{\Gamma\left(1+\gamma_{1}\right)}\right)\right. \\
& \left.+\frac{\left\|\mu_{2}\right\|}{\Gamma(\alpha+1)}\left(1+\frac{1}{\Gamma\left(2-\beta_{2}\right)}+\frac{1}{\Gamma\left(1+\gamma_{2}\right)}\right)\right]
\end{aligned}
$$

and

$$
\begin{aligned}
\left|(B u)^{\prime}(t)-(B v)^{\prime}(t)\right|= & \mid \frac{1}{\Gamma(\alpha-1)} \int_{0}^{t_{0}}(t-s)^{\alpha-2} \\
& \times f_{1}\left(s, u(s),{ }^{c} D^{\beta_{1}} u(s), I^{\gamma_{1}} u(s)\right) d s \\
& +\frac{1}{\Gamma(\alpha-1)} \int_{t_{0}}^{t}(t-s)^{\alpha-2}
\end{aligned}
$$




$$
\begin{aligned}
& \times f_{2}\left(s, u(s),{ }^{c} D^{\beta_{2}} u(s), I^{\gamma_{2}} u(s)\right) d s \\
- & \frac{1}{\Gamma(\alpha-1)} \int_{0}^{t_{0}}(t-s)^{\alpha-2} \\
\times & f_{1}\left(s, v(s),{ }^{c} D^{\beta_{1}} v(s), I^{\gamma_{1}} v(s)\right) d s \\
& -\frac{1}{\Gamma(\alpha-1)} \int_{t_{0}}^{t}(t-s)^{\alpha-2} \\
& \times f_{2}\left(s, v(s),{ }^{c} D^{\beta_{2}} v(s), I^{\gamma_{2}} v(s)\right) d s \mid \\
\leq & \|u-v\|_{*}\left[\frac{\left\|\mu_{1}\right\|}{\Gamma(\alpha)}\left(1+\frac{1}{\Gamma\left(2-\beta_{1}\right)}+\frac{1}{\Gamma\left(1+\gamma_{1}\right)}\right)\right. \\
& \left.+\frac{\left\|\mu_{2}\right\|}{\Gamma(\alpha)}\left(1+\frac{1}{\Gamma\left(2-\beta_{2}\right)}+\frac{1}{\Gamma\left(1+\gamma_{2}\right)}\right)\right] .
\end{aligned}
$$

Therefore,

$$
\begin{aligned}
\|B u-B v\|_{*} \leq & {\left[\frac{\left\|\mu_{1}\right\|}{\Gamma(\alpha)}\left(1+\frac{1}{\Gamma\left(2-\beta_{1}\right)}+\frac{1}{\Gamma\left(1+\gamma_{1}\right)}\right)\right.} \\
& \left.+\frac{\left\|\mu_{2}\right\|}{\Gamma(\alpha)}\left(1+\frac{1}{\Gamma\left(2-\beta_{2}\right)}+\frac{1}{\Gamma\left(1+\gamma_{2}\right)}\right)\right]\left(\frac{1}{\alpha}+1\right)\|u-v\|_{*} \\
\leq & \Delta\|u-v\|_{*} .
\end{aligned}
$$

Since $\Delta<1$, therefore $B$ is a contraction. Hence, all the conditions of Theorem 6 are satisfied, and there exists $x \in S$ such that $A x+B x=x$. Thus, equation (1) has a solution on $J$. This completes the proof.

Example 1 Consider the following fractional differential equation:

$$
{ }^{c} D^{\frac{3}{2}} u(t)=\left\{\begin{array}{cc}
\frac{t^{2}+\frac{1}{2} t-\frac{1}{2}}{100}\left[u(t)+\tan ^{-1}\left({ }^{c} D^{\frac{1}{3}} u(t)\right)\right. & \\
\left.+\sin \left(I^{\sqrt{2}} u(t)\right)\right], & 0 \leq t \leq \frac{1}{2}, \\
\frac{t^{2}+\frac{(\sqrt{2}-1)}{2} t-\frac{\sqrt{2}}{4}}{100}\left[\frac{|u(t)|}{1+|x(t)|}\right. & \\
\left.+\frac{\left.\right|^{c} D^{\frac{1}{4}} u(t)+I^{\sqrt{3}} u(t) \mid}{1+\left.\right|^{c} D^{\frac{1}{4}} u(t)+I^{\sqrt{3}} u(t) \mid}\right], & \frac{1}{2} \leq t \leq 1,
\end{array}\right.
$$

with boundary conditions

$$
u(0)=\frac{e^{\frac{1}{2}}}{100}\left[\frac{\left|u\left(\frac{1}{2}\right)+{ }^{c} D^{\frac{1}{5}} x\left(\frac{1}{2}\right)+I^{\sqrt{5}} u\left(\frac{1}{2}\right)\right|}{1+\left|u\left(\frac{1}{2}\right)+{ }^{c} D^{\frac{1}{5}} u\left(\frac{1}{2}\right)+I^{\sqrt{5}} u\left(\frac{1}{2}\right)\right|}\right]
$$

and

$$
\begin{aligned}
u(1)= & \frac{1}{100} \sin \left(\frac{1}{2}\right)\left[\cos \left(u\left(\frac{1}{2}\right)\right)+\sin \left({ }^{c} D^{\frac{1}{6}} u\left(\frac{1}{2}\right)\right)\right. \\
& \left.+\tan ^{-1}\left(I^{\sqrt{6}} u\left(\frac{1}{2}\right)\right)\right] .
\end{aligned}
$$


Here, $\alpha=\frac{3}{2}, \beta_{1}=\frac{1}{3}, \beta_{2}=\frac{1}{4}, \beta_{3}=\frac{1}{5}, \beta_{4}=\frac{1}{6}, \gamma_{1}=\sqrt{2}, \gamma_{2}=\sqrt{3}, \gamma_{3}=\sqrt{5}, \gamma_{4}=\sqrt{6}, t_{0}=\frac{1}{2}$,

$$
\begin{aligned}
& f_{1}\left(t, x_{1}, x_{2}, x_{3}\right)=\frac{t^{2}+\frac{1}{2} t-\frac{1}{2}}{100}\left(x_{1}+\tan ^{-1} x_{2}+\sin x_{3}\right), \\
& f_{2}\left(t, x_{1}, x_{2}, x_{3}\right)=\frac{t^{2}+\frac{(\sqrt{2}-1)}{2} t-\frac{\sqrt{2}}{4}}{100}\left(\frac{\left|x_{1}\right|}{1+\left|x_{1}\right|}+\frac{\left|x_{2}+x_{3}\right|}{1+\left|x_{2}+x_{3}\right|}\right), \\
& h_{1}\left(t, x_{1}, x_{2}, x_{3}\right)=\frac{e^{t}}{100}\left(\frac{\left|x_{1}+x_{2}+x_{3}\right|}{1+\left|x_{1}+x_{2}+x_{3}\right|}\right), \\
& h_{2}\left(t, x_{1}, x_{2}, x_{3}\right)=\frac{1}{100} \sin (t)\left(\cos \left(x_{1}\right)+\sin \left(x_{2}\right)+\tan ^{-1}\left(x_{3}\right)\right) .
\end{aligned}
$$

Clearly,

$$
\begin{aligned}
& \left|f_{1}\left(t, x_{1}, x_{2}, x_{3}\right)-f_{1}\left(t, x_{1}^{\prime}, x_{2}^{\prime}, x_{3}^{\prime}\right)\right| \leq \frac{1}{100} \sum_{j=1}^{3}\left|x j-x_{j}^{\prime}\right|, \\
& \left|f_{2}\left(t, x_{1}, x_{2}, x_{3}\right)-f_{2}\left(t, x_{1}^{\prime}, x_{2}^{\prime}, x_{3}^{\prime}\right)\right| \leq \frac{2+\sqrt{2}}{400} \sum_{j=1}^{3}\left|x j-x_{j}^{\prime}\right|, \\
& \left|h_{1}\left(t, x_{1}, x_{2}, x_{3}\right)-h_{1}\left(t, x_{1}^{\prime}, x_{2}^{\prime}, x_{3}^{\prime}\right)\right| \leq \frac{1}{100} e \sum_{j=1}^{3}\left|x j-x_{j}^{\prime}\right|, \\
& \left|h_{2}\left(t, x_{1}, x_{2}, x_{3}\right)-h_{2}\left(t, x_{1}^{\prime}, x_{2}^{\prime}, x_{3}^{\prime}\right)\right| \leq \frac{1}{100} \sin (1) \sum_{j=1}^{3}\left|x j-x_{j}^{\prime}\right|
\end{aligned}
$$

for all $t \in \bar{J}$ and $x_{j}, x_{j}^{\prime} \in \mathbb{R}$. Hence, $l_{1}=\frac{1}{100}, l_{2}=\frac{2+\sqrt{2}}{400}, l_{3}=\frac{1}{100} e, l_{4}=\frac{1}{100}$, and

$$
\begin{aligned}
& \frac{3 l_{1}}{\Gamma(\alpha+1)}\left[1+\frac{1}{\Gamma\left(2-\beta_{1}\right)}+\frac{1}{\Gamma\left(1+\gamma_{1}\right)}\right] \\
& +\frac{3 l_{2}}{\Gamma(\alpha+1)}\left[1+\frac{1}{\Gamma\left(2-\beta_{2}\right)}+\frac{1}{\Gamma\left(1+\gamma_{2}\right)}\right] \\
& +3 l_{3}\left[1+\frac{1}{\Gamma\left(2-\beta_{3}\right)}+\frac{1}{\Gamma\left(1+\gamma_{3}\right)}\right] \\
& +2 l_{4}\left[1+\frac{1}{\Gamma\left(2-\beta_{4}\right)}+\frac{1}{\Gamma\left(1+\gamma_{4}\right)}\right] \\
& +\frac{l_{1}}{\Gamma(\alpha)}\left[1+\frac{1}{\Gamma\left(2-\beta_{1}\right)}+\frac{1}{\Gamma\left(1+\gamma_{1}\right)}\right] \\
& \quad+\frac{l_{2}}{\Gamma(\alpha)}\left[1+\frac{1}{\Gamma\left(2-\beta_{2}\right)}+\frac{1}{\Gamma\left(1+\gamma_{2}\right)}\right] \\
& \simeq 0.4872<1 .
\end{aligned}
$$

Therefore, all the conditions of Corollary 2 are satisfied and equation 7 with boundary conditions (8) and (9) has the unique solution on $J$. 
Example 2 Consider the following fractional boundary value problem:

$$
{ }^{c} D^{\frac{3}{2}} u(t)= \begin{cases}\frac{\ln \left(t+\frac{3}{4}\right)}{2 t+\pi^{2}+2}\left[\frac{\left|u(t)+{ }^{c} D^{\frac{1}{5}} u(t)+I^{\frac{1}{3}} u(t)\right|}{1+\left|u(t)+{ }^{c} D^{\frac{1}{5}} u(t)+I^{\frac{1}{3}} u(t)\right|}\right], & 0 \leq t \leq \frac{1}{4}, \\ \frac{1}{e^{2}+1}\left(t-\frac{1}{4}\right)^{2}\left[x(t)+\cos \left({ }^{c} D^{\frac{1}{5}} x(t)\right)\right. & \\ \left.\quad+\sin \left(I^{\frac{2}{3}} x(t)\right)\right], & \frac{1}{4} \leq t \leq 1,\end{cases}
$$

with boundary conditions

$$
u(0)=e^{\frac{1}{4}}\left[u\left(\frac{1}{4}\right)+{ }^{c} D^{\frac{3}{5}} u\left(\frac{1}{4}\right)+I^{\frac{4}{3}} u\left(\frac{1}{4}\right)\right]
$$

and

$$
u(1)=\sin \left(\frac{1}{4}\right)\left[u\left(\frac{1}{4}\right)+{ }^{c} D^{\frac{4}{5}} u\left(\frac{1}{4}\right)+I^{\frac{5}{3}} u\left(\frac{1}{4}\right)\right]^{\frac{1}{2}} .
$$

Here, $\alpha=\frac{4}{3}, \beta_{1}=\frac{1}{5}, \beta_{2}=\frac{2}{5}, \beta_{3}=\frac{3}{5}, \beta_{4}=\frac{4}{5}, \gamma_{1}=\frac{1}{3}, \gamma_{2}=\frac{2}{3}, \gamma_{3}=\frac{4}{3}, \gamma_{4}=\frac{5}{3}, t_{0}=\frac{1}{4}$,

$$
\begin{aligned}
& f_{1}\left(t, x_{1}, x_{2}, x_{3}\right)=\frac{\ln \left(t+\frac{3}{4}\right)}{2 t+\pi^{2}+2}\left(\frac{\left|x_{1}+x_{2}+x_{3}\right|}{1+\left|x_{1}+x_{2}+x_{3}\right|}\right), \\
& f_{2}\left(t, x_{1}, x_{2}, x_{3}\right)=\frac{1}{e^{2}+1}\left(t-\frac{1}{4}\right)^{2}\left(x_{1}+x_{2}+x_{3}\right), \\
& h_{1}\left(t, x_{1}, x_{2}, x_{3}\right)=e^{t}\left(x_{1}+x_{2}+x_{3}\right), \\
& h_{2}\left(t, x_{1}, x_{2}, x_{3}\right)=\sin (t)\left(x_{1}+x_{2}+x_{3}\right)^{\frac{1}{2}} .
\end{aligned}
$$

Since each function with boundary derivative has a Lipschitz condition, the map $f(x)=$ $\frac{|x|}{1+|x|}$ is Lipschitz. Hence, it is clear that

$$
\begin{aligned}
& \left|f_{1}\left(t, x_{1}, x_{2}, x_{3}\right)-f_{1}\left(t, x_{1}^{\prime}, x_{2}^{\prime}, x_{3}^{\prime}\right)\right| \leq \frac{\ln \left(t+\frac{3}{4}\right)}{2 t+\pi^{2}+2} \sum_{j=1}^{3}\left|x_{j}-x_{j}^{\prime}\right|, \\
& \left|f_{2}\left(t, x_{1}, x_{2}, x_{3}\right)-f_{2}\left(t, x_{1}^{\prime}, x_{2}^{\prime}, x_{3}^{\prime}\right) \leq \frac{1}{e^{2}+1}\left(t-\frac{1}{4}\right)^{2} \sum_{j=1}^{3}\right| x_{j}-x_{j}^{\prime} \mid, \\
& \left|h_{1}\left(t, x_{1}, x_{2}, x_{3}\right)\right| \leq e^{t} \sum_{j=1}^{3}\left|x_{j}\right|, \\
& \left|h_{2}\left(t, x_{1}, x_{2}, x_{3}\right)\right| \leq \sin (t)\left[\sum_{j=1}^{3}\left|x_{j}\right|\right]^{\frac{1}{2}}
\end{aligned}
$$

for all $t \in \bar{J}$ and $x_{j}, x_{j}^{\prime} \in \mathbb{R}$. By choosing

$$
\mu_{1}(t)=\frac{\ln \left(t+\frac{3}{4}\right)}{2 t+\pi^{2}+2}, \quad \mu_{2}(t)=\frac{1}{e^{2}+1}\left(t-\frac{1}{4}\right)^{2}
$$


$v_{1}(t)=e^{t}, v_{2}(t)=\sin (t), \psi_{1}(t)=t$, and $\psi_{2}(t)=t^{\frac{1}{2}}$, we get

$$
\begin{aligned}
\Delta= & {\left[\frac{\left\|\mu_{1}\right\|}{\Gamma(\alpha)}\left(1+\frac{1}{\Gamma\left(2-\beta_{1}\right)}+\frac{1}{\Gamma\left(1+\gamma_{1}\right)}\right)\right.} \\
& \left.+\frac{\left\|m u_{2}\right\|}{\Gamma(\alpha)}\left(1+\frac{1}{\Gamma\left(2-\beta_{2}\right)}+\frac{1}{\Gamma\left(1+\gamma_{2}\right)}\right)\right]\left(\frac{1}{\alpha}+1\right)
\end{aligned}
$$

$\simeq 0.9484<1$

Therefore, all the conditions of Theorem 10 are satisfied and equation (10) with boundary conditions (11) and (12) has a solution on $J$.

\section{Funding}

Not applicable.

\section{Availability of data and materials}

Not applicable.

\section{Ethics approval and consent to participate}

Not applicable.

\section{Competing interests}

The authors declare that they have no competing interests.

\section{Consent for publication}

Not applicable.

\section{Authors' contributions}

All authors contributed equally and significantly in this manuscript, and they read and approved the final manuscript.

\section{Author details}

${ }^{1}$ Department of Mathematics, Azarbaijan Shahid Madani University, Tabriz, Iran. ${ }^{2}$ Department of Mathematics, Shahid Mehrab Madani Educational Institution, Hamedan, Iran. ${ }^{3}$ Department of Mathematics, Faculty of Science, Bu-Ali Sina University, Hamedan, Iran.

\section{Publisher's Note}

Springer Nature remains neutral with regard to jurisdictional claims in published maps and institutional affiliations.

Received: 11 July 2019 Accepted: 13 August 2019 Published online: 28 August 2019

\section{References}

1. Kilbas, A.A., Srivastava, H.M., Trujillo, J.J.: Theory and Applications of Fractional Differential Equations. Elsevier, Amsterdam (2006)

2. Samko, S.G., Kilbas, A.A., Marichev, O.I.: Fractional Integrals and Derivatives: Theory and Applications. Gordon \& Breach, Philadelphia (1993)

3. Podlubny, I.: Fractional Differential Equations. Academic Press, San Diego (1999)

4. Kac, V., Cheung, P.: Quantum Calculus. Universitext. Springer, New York (2002)

5. Su, X., Zhang, S.: Solutions to boundary value problems for nonlinear differential equations of fractional order. Electron. J. Differ. Equ. 2009(26), 1 (2009)

6. Ahmad, B., Sivasundaram, S.: On four-point nonlocal boundary value problems of nonlinear integro-differential equations of fractional order. J. Appl. Math. Comput. 217(2), 480-487 (2010). https://doi.org/10.1016/j.amc.2010.05.080

7. Agarwal, R.P., O'Regan, D., Staněk, S.: The existence of solutions for a nonlinear mixed problem of singular fractional differential equations. Math. Nachr. 285(1), 27-41 (2012). https://doi.org/10.1002/mana.201000043

8. Baleanu, D., Rezapour, S., Mohammadi, H.: Some existence results on nonlinear fractional differential equations. Philos. Trans. R. Soc., Math. Phys. Eng. Sci. 2013, 371 (2013). https://doi.org/10.1098/rsta.2012.0144

9. Baleanu, D., Mohammadi, H., Rezapour, S.: The existence of solutions for a nonlinear mixed problem of singular fractional differential equations. Adv. Differ. Equ. 2013, 359 (2013). https://doi.org/10.1186/1687-1847-2013-359

10. Baleanu, D., Nazemi, S.Z., Rezapour, S.: Existence and uniqueness of solutions for multi-term nonlinear fractional integro-differential equations. Adv. Differ. Equ. 2013(1), 368 (2013). https://doi.org/10.1186/1687-1847-2013-368

11. Agarwal, R.P., Baleanu, D., Hedayati, V., Rezapour, S.: Two fractional derivative inclusion problems via integral boundary condition. Appl. Math. Comput. 257, 205-212 (2015). https://doi.org/10.1016/j.amc.2014.10.082

12. Zhang, X., Zhong, Q.: Multiple positive solutions for nonlocal boundary value problems of singular fractional differential equations. Bound. Value Probl. 2016, 65 (2016). https://doi.org/10.1186/s13661-016-0572-0 
13. Rezapour, S., Hedayati, V.: On a Caputo fractional differential inclusion with integral boundary condition for convex-compact and nonconvex-compact valued multifunctions. Kragujev. J. Math. 41(1), 143-158 (2017). https://doi.org/10.5937/KgJMath1701143R

14. Aydogan, M.S., Baleanu, D., Mousalou, A., Rezapour, S.: On high order fractional integro-differential equations including the Caputo-Fabrizio derivative. Bound. Value Probl. 2018(1), 90 (2018). https://doi.org/10.1186/s13661-018-1008-9

15. Baleanu, D., Mousalou, A., Rezapour, S.: The extended fractional Caputo-Fabrizio derivative of order $0 \leq \sigma<1$ on c $[0,1]$ and the existence of solutions for two higher-order series-type differential equations. Adv. Differ. Equ. 2018(1), 255 (2018). https://doi.org/10.1186/s13662-018-1696-6

16. Samei, M.E., Khalilzadeh Ranjbar, G.: Some theorems of existence of solutions for fractional hybrid $q$-difference inclusion. J. Adv. Math. Stud. 12(1), 63-76 (2019)

17. Ahmad, B., Ntouyas, S.K., Purnaras, I.K.: Existence results for nonlocal boundary value problems of nonlinear fractional q-difference equations. Adv. Differ. Equ. 2012, 140 (2012). https://doi.org/10.1186/1687-1847-2012-140

18. Ahmad, B., Nieto, J.J.: Riemann-Liouville fractional integro-differential equations with fractional nonlocal integral boundary conditions. Bound. Value Probl. 2011, 36 (2011). https://doi.org/10.1186/1687-2770-2011-36

19. Baleanu, D., Hedayati, V., Rezapour, S., Al Qurashi, M.M.: On two fractional differential inclusions. SpringerPlus 5(1), 882 (2016). https://doi.org/10.1186/s40064-016-2564-z

20. Agarwal, R.P., Belmekki, M., Benchohra, M.: A survey on semilinear differential equations and inclusions involving Riemann-Liouville fractional derivative. Adv. Differ. Equ. 2009, 981728 (2009). https://doi.org/10.1155/2009/981728

21. Baleanu, D., Agarwal, R.P., Mohammadi, H., Rezapour, S.: Some existence results for a nonlinear fractional differential equation on partially ordered Banach spaces. Bound. Value Probl. 2013, 112 (2013). https://doi.org/10.1186/1687-2770-2013-112

22. Anastassiou, G.A.: Principles of delta fractional calculus on time scales and inequalities. Math. Comput. Model. $\mathbf{5 2}$ 556-566 (2010). https://doi.org/10.1016/j.mcm.2010.03.055

23. Agarwal, R.P., Ahmad, B.: Existence theory for anti-periodic boundary value problems of fractional differential equations and inclusions. Comput. Math. Appl. 62, 1200-1214 (2011). https://doi.org/10.1016/j.camwa.2011.03.001

24. Liu, X., Liu, Z.: Existence result for fractional differential inclusions with multivalued term depending on lower-order derivative. Abstr. Appl. Anal. 2012, 24 (2012). https://doi.org/10.1155/2012/423796

25. Abdeljawad, T., Alzabut, J., Baleanu, D.: A generalized q-fractional Gronwall inequality and its applications to non-linear delay q-fractional difference systems. J. Inequal. Appl. 2016, 240 (2016). https://doi.org/10.1186/s13660-016-1181-2

26. Ragusa, M.A.: Local Hölder regularity for solutions of elliptic systems. Duke Math. J. 113(2), 385-397 (2002)

27. Ragusa, M.A.: Cauchy-Dirichlet problem associated to divergence form parabolic equations. Commun. Contemp. Math. 6(3), 377-393 (2004). https://doi.org/10.1142/S0219199704001392

28. Bachar, I., Mâagli, H., Rădulescu, V.D.: Fractional Navier boundary value problems. Bound. Value Probl. 2016(79), 14 (2016). https://doi.org/10.1186/s13661-016-0586-7

29. Denton, Z., Ramírez, J.D.: Existence of minimal and maximal solutions to RL fractional integro-differential initial value problems. Opusc. Math. 37(5), 705-724 (2017). https://doi.org/10.7494/OpMath.2017.37.5.705

30. Chidouh, A., Torres, D.: Existence of positive solutions to a discrete fractional boundary value problem and corresponding Lyapunov-type inequalities. Opusc. Math. 38(1), 31-40 (2018). https://doi.org/10.7494/OpMath.2018.38.1.31

31. Liu, Y.: A new method for converting boundary value problems for impulsive fractional differential equations to integral equations and its applications. Adv. Nonlinear Anal. 8(1), 386-454 (2019). https://doi.org/10.1515/anona-2016-0064

32. Smart, D.R.: Fixed Point Theorems. Cambridge University Press, New York (1980)

\section{Submit your manuscript to a SpringerOpen ${ }^{\circ}$ journal and benefit from:}

- Convenient online submission

- Rigorous peer review

- Open access: articles freely available online

- High visibility within the field

- Retaining the copyright to your article

Submit your next manuscript at $\boldsymbol{s p r i n g e r o p e n . c o m ~}$ 